\title{
Radar Coincidence Imaging for Off-Grid Target Using Frequency-Hopping Waveforms
}

\author{
Xiaoli Zhou, Hongqiang Wang, Yongqiang Cheng, Yuliang Qin, and Haowen Chen
}

School of Electronic Science and Engineering, National University of Defense Technology, Changsha 410073, China

Correspondence should be addressed to Xiaoli Zhou; zhouxiaoli@nudt.edu.cn

Received 8 November 2015; Accepted 14 March 2016

Academic Editor: Wen-Qin Wang

Copyright ( 2016 Xiaoli Zhou et al. This is an open access article distributed under the Creative Commons Attribution License, which permits unrestricted use, distribution, and reproduction in any medium, provided the original work is properly cited.

\begin{abstract}
Radar coincidence imaging (RCI) is a high-resolution staring imaging technique without the limitation of the target relative motion. To achieve better imaging performance, sparse reconstruction is commonly used. While its performance is based on the assumption that the scatterers are located at the prediscretized grid-cell centers, otherwise, off-grid emerges and the performance of RCI degrades significantly. In this paper, RCI using frequency-hopping (FH) waveforms is considered. The off-grid effects are analyzed, and the corresponding constrained Cramér-Rao bound (CCRB) is derived based on the mean square error (MSE) of the "oracle" estimator. For off-grid RCI, the process is composed of two stages: grid matching and off-grid error (OGE) calibration, where twodimension (2D) band-excluded locally optimized orthogonal matching pursuit (BLOOMP) and alternating iteration minimization (AIM) algorithms are proposed, respectively. Unlike traditional sparse recovery methods, BLOOMP realizes the recovery in the refinement grids by overwhelming the shortages of coherent dictionary and is robust to noise and OGE. AIM calibration algorithm adaptively adjusts the OGE and, meanwhile, seeks the optimal target reconstruction result.
\end{abstract}

\section{Introduction}

Radar coincidence imaging (RCI), originated from the optical coincidence imaging, is a staring imaging technique which can obtain focused high-resolution image without the limitation of the target relative motion [1-3]. RCI can operate under the nonideal observing geometry of forwardlooking/staring, with significant potentials for resolution enhancement, interference, and jamming suppression. The essential principle of RCI is to produce time-space independent signals in the imaging area, while the frequency-hopping (FH) waveforms are good candidates because they are easily generated and have constant modulus $[4,5]$. Besides, comparing with the linear frequency modulated (LFM) waveforms which are often used in the traditional radar systems, FH waveforms can suppress the range ambiguity, decouple the range and Doppler, and are also attractive for their merits on electronic counter-countermeasures (ECCM) and reducing interference between adjacent radar systems for sharing the frequency spectrum [6]. Hence, we focus on RCI using FH waveforms (FH-RCI) in this paper.

In RCI, the continuous target space needs to be discretized to a fine grid and the target-scattering centers are assumed to be exactly located at these prediscretized grid-cell centers [3]. Then, the detecting signals at different grid-cell centers can be formed as the atoms of sparse representation dictionary. Meanwhile, the scatterers of target are often distributed sparsely in most radar imaging applications. Thus, sparse recovery approaches and compressive sensing (CS) $[7,8]$ are suitable for RCI by exploiting the sparsity of target in the target space. In the sparse reconstruction theory, signal reconstruction depends on presetting an appropriate sparsifying dictionary which is supported on the assumed grids and defines the signal sparsity. However, as the scatterers are distributed in a continuous scene, the scattering centers are generally located off the grid-cell centers, no matter how fine the grid is; therefore, off-grid yields [9]. Then, the performance of RCI would be severely affected.

Off-grid would lead to the mismatch between the assumed and actual sparsifying dictionaries directly, which causes the performance of conventional sparse recovery methods to degrade considerably [10-13]. Intuitively speaking, the sparse elements in the signal may not lie on the assumed grids and not perfectly match the dictionary; thus, the true signal is not exactly supported on the assumed 
dictionary. Moreover, the signal recovery is robust to the mismatch in the sense that the recovery error grows with the mismatch level and is independent of the sparsity of the original signal. Thus, the sparse reconstruction performance of radar imaging degrades severely [3, 9, 14-17].

Considering the off-grid, several algorithms have been proposed. One simple approach is to use multiresolution refinement strategy and decrease the grid size iteratively [18]. Nevertheless, a finer grid may enhance the coherence between the columns of dictionary and increase the computational complexity and numerical instability of reconstruction [17]. Modeling the off-grid as a multiplicative perturbation, the sparse total least squares (S-TLS) [19] and joint correlation-parameterization (CP) [3] algorithms are proposed. However, the algorithms are inefficient without considering possibly available a priori information and the performance degrades rapidly when the off-grid is significant. To explore the structure of dictionary mismatch, the support-constrained orthogonal matching pursuit (SCOMP) [16] and joint sparse signal recovery methods [20] are proposed based on the first-order Taylor expansion to utilize the support constraint, while the methods eventually break down when the gridding error dominates the data. Lately, from the sparse Bayesian learning (SBL) perspective, several approaches are proposed, such as off-grid sparse Bayesian inference (OGSBI) [21], sparse adaptive calibration recovery via iterative maximum a posteriori (SACR-iMAP) [9], and variational expectation-maximization $[10,22]$ algorithms, to achieve joint sparse recovery. In the SBL framework, the sparsity is exploited in the signal of interest. The merit of SBL is its flexibility in modeling sparse signals that can not only promote the sparsity of its solution but also exploit the possible structure of the signal to be recovered [21], whereas it offers few guarantees on the signal recovery accuracy.

Another way to sidestep the off-grid is to work directly on the continuous parameter space. An atomic norm minimization approach [23], which yields an infinite dictionary of continuous atoms and arbitrarily high coherence, is proposed to exactly identify the unknown parameters directly. In [24], the continuous basis pursuit (CBP), which uses a dictionary with an auxiliary interpolation function to overcome the off-grid, is proposed to overcome the limitation of BP. However, any noise will make the exact results unidentifiable, and the computational burden and numerical instability are significant.

In conclusion, there are variable methods to solve the off-grid. However, most algorithms provide no performance guarantees on signal recovery and the performance deteriorates significantly when the off-grid error (OGE) increases. Furthermore, most approaches cannot be applied to off-grid RCI directly, as they are proposed for DOA or spectral estimation applications without considering the specific problems existing in RCI, such as the coupling among the parameters in 2D/3D imaging. Moreover, the computational complexity increases significantly in $2 \mathrm{D} / 3 \mathrm{D}$ case.

Thus, we investigate the off-grid FH-RCI in this paper and present an off-grid imaging approach. The main contributions of this paper are as follows. (a) The off-grid effect is investigated. The off-grid FH-RCI model is derived and the methodology to analyze the offgrid is established. The relative imaging error (RIE), gridding error, and signal-error-ratio (SER) are introduced to model the OGE. The gridding error is seriously sensitive to the OGE and induces the imaging quality to degrade drastically. Furthermore, the corresponding constrained Cramér-Rao bound (CCRB) is also derived to analyze the off-grid effect.

(b) A novel sparse recovery approach for off-grid RCI is presented. Off-grid RCI is a nonconvex optimization problem and can be solved by two stages. (1) The first is grid matching; that is, the scatterers are captured by the closest grid-cells. The band-excluded locally optimized orthogonal matching pursuit (BLOOMP) approach [25] is introduced and extended to $2 \mathrm{D}$ version which is operated on range-azimuth space for RCI. (2) OGE calibration, namely, estimate the OGE between the actual scatterer location and its closest grid-cell center. The sparse alternating iteration minimization (AIM) approach is used. Numerical experiments show that the proposed method realizes the target reconstruction robustly and achieves both high-resolution and outstanding imaging quality and is also simple to implement.

The rest of the paper is organized as follows. Section 2 presents the off-grid FH-RCI model in the range-azimuth space. Section 3 investigates the off-grid effects by both numerical simulations and theoretical derivations. Then, the image reconstruction method is proposed in Section 4. In Section 5, some numerical examples are given to verify the performance of the presented method. Finally, some comments and conclusions are shown in Section 6.

A comment on notation: we use boldface lowercase letters for vectors and boldface uppercase letters for matrices. $(\cdot)^{T}$, $(\cdot)^{-1}$, and $(\cdot)^{\dagger}$ denote the transpose, inverse, and pseudoinverse of a matrix, respectively. $\operatorname{diag}(\cdot), \odot$, and $\operatorname{vec}(\cdot)$ are the diagonalization, Hadamard product, and vectorization operation, separately. Finally, $\|\cdot\|_{2}$ denotes the Euclidean norm of a vector.

\section{Problem Formulation}

2.1. Signal Model. RCI can be realized by a multiple-input multiple-output (MIMO) radar system to transmit timeindependent and group-orthogonal signals [1]. Thus, the spatial variety of wavefront increases, and the scatterers within a beam then reflect different detecting signals according to their respective locations. So the superresolution within the beam emerges. Compared with conventional MIMO radar which focuses on multiple paths or multiple observation angles, RCI needs the interference of transmitted waveforms to make the wavefront show spatial fluctuation and increase the spatial variety of detecting signals. Besides, the components of each path are separated utilizing the waveform orthogonality in conventional MIMO radar, while the components are not separated in the whole RCI procedure.

In this paper, a RCI system with $M_{T}$ transmitters and $M_{R}$ receivers is considered; each transmitter emits an independent $\mathrm{FH}$ waveform. Assume that both transmitters and receivers are configured as a uniform linear array (ULA) 


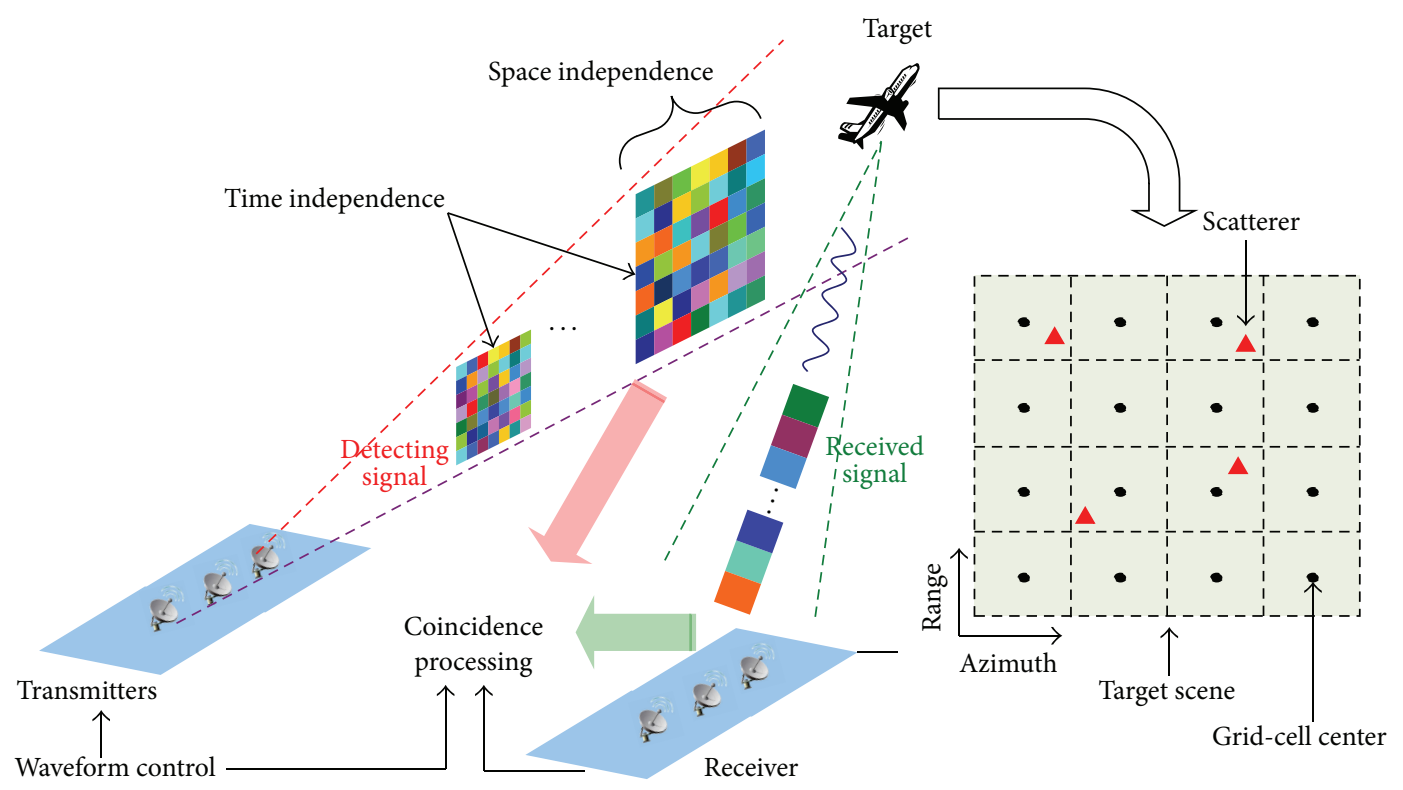

Figure 1: Geometry of off-grid RCI.

and the interelement spacings of the transmit and receive antennas is $d_{T}$ and $d_{R}$, respectively. The $m$ th transmitter emits constant modulus FH waveform $s_{m}(t)$. Assuming $L$ pulses comprise a waveform, the signal from the $m$ th transmitter is [4]

$$
s_{m}(t)=\sum_{l=0}^{L-1} \phi_{m}(t-l T)
$$

where $\phi_{m}(t)=\sum_{q=0}^{Q-1} e^{j 2 \pi c_{m, q} \Delta f t} u(t-q \Delta t)$ and

$$
u(t) \triangleq \begin{cases}1 & 0<t<\Delta t \\ 0 & \text { otherwise }\end{cases}
$$

where $T, \Delta t$, and $\Delta f$ denote the pulse repetition interval (PRI), FH duration, and $\mathrm{FH}$ interval, respectively. $c_{m, q} \epsilon$ $\{1, \ldots, G\}$ is the $\mathrm{FH}$ code which specifies the transmitted frequency during each hopping interval, where $G$ is a positive integer. $Q$ is the length of the code. Thus, the PRI is $T=Q \Delta t$.

To ensure the orthogonality of waveforms, for each hopping interval, the codes are assumed to be constrained to satisfy [5]

$$
c_{m, q} \neq c_{m^{\prime}, q}, \text { for } m \neq m^{\prime}, \forall q
$$

$c_{m, q}$ can be arranged into an $M \times Q$ dimensional code matrix specifying the transmitted frequencies.

Without loss of generality, the target is assumed to consist of several ideal point scattering centers for an enough high carrier frequency, which is widely used in the imaging radar system. Furthermore, there are $P$ scatterers which are widely separated to provide some performance guarantee.

The target scene is considered to be a $2 \mathrm{D}$ range-azimuth space as illustrated in Figure 1. RCI discretizes the continuous target scene and generates a number of grid points, and the scatterers are assumed to be located at the grid-cell centers. Thus, the scene is discretized with $U$ azimuth bins, $V$ range bins, and associated bin sizes $\Delta_{\theta}$ and $\Delta_{R}$. Hence, the number of grid-cells is $K=U V$. The $k$ th scatterer is located at the grid-cell center $\left(\theta_{k}, R_{k}\right)=\left(\theta_{0}+u_{(k)} \Delta_{\theta}, R_{0}+v_{(k)} \Delta_{R}\right)$, where $\theta_{0}$ and $R_{0}$ are reference values in the respective domains and the pair $\left(u_{(k)}, v_{(k)}\right)$ represents the $k$ th grid-cell in the discretized scene. As the scatterers possess nonzero scattering coefficient $\beta_{k}$ which is proportional to the radar cross section (RCS), the associated $\beta_{k}=0$ means that there is no scatterer at the $k$ th grid-cell center. Denote by $S=\left\{k \in\{1,2, \ldots, K\}: \beta_{k} \neq 0\right\}$ the index set of scatterer location.

The received demodulated baseband echo is a linear combination of all the scatterers reflected waveforms from all the transmitters. Although contributions to the echo only result from the $P$ scatterers, it is unknown a priori which $\beta_{k}$ is nonzero. Thus, we sum over all $K$ possible locations, and the received baseband signal at the $n$th receiver can be expressed as

$$
y_{n}(t)=\sum_{m=1}^{M_{T}} \sum_{l=0}^{L-1} \sum_{q=0}^{Q-1} \sum_{k \in S} \beta_{k} e^{j 2 \pi c_{m, q} \Delta f\left(t-l T-\tau_{m, n}^{k}\right)} u\left(t-q \Delta t-l T-\tau_{m, n}^{k}\right) e^{-j 2 \pi f_{c}\left(l T+\tau_{m, n}^{k}\right)}+w_{n}(t)
$$


where $f_{c}$ is the carrier frequency. $w_{n}$, an independent complex Gaussian random process with zero-mean and variance $\sigma^{2}$, denotes the noise at the receiver. $\tau_{m, n}^{k}$, defined as (5), is the relative propagation delay corresponding to the $m$ th transmitter and the $n$th receiver with respect to the $k$ th gridcell center, where $c$ is the speed of wave propagation:

$$
\tau_{m, n}^{k}=\frac{1}{c}\left(d_{m, k}^{(T)}+d_{n, k}^{(R)}\right),
$$

$$
\begin{aligned}
& d_{m, k}^{(T)}=\sqrt{\left(R_{k} \sin \theta_{k}+(m-1) d_{T}\right)^{2}+\left(R_{k} \cos \theta_{k}\right)^{2}}, \\
& d_{n, k}^{(R)}=\sqrt{\left(R_{k} \sin \theta_{k}+(n-1) d_{R}\right)^{2}+\left(R_{k} \cos \theta_{k}\right)^{2}} .
\end{aligned}
$$

Then, we sample the received signal with the interval $t_{s}$ and obtain $N$ samples. After that, stack $\left\{y_{n}(1), \ldots, y_{n}(N)\right\}_{n=1}^{M_{R}}$ and $\left\{w_{n}(1), \ldots, w_{n}(N)\right\}_{n=1}^{M_{R}}$ into column vectors $\mathbf{y}$ and $\mathbf{w}$, respectively, via the vectorization operation $\operatorname{vec}(\cdot)$,

$$
\mathbf{A}_{k}=\operatorname{vec}\left(\sum_{m=1}^{M_{T}} \sum_{l=0}^{L-1} \sum_{q=0}^{Q-1} e^{j 2 \pi c_{m, q} \Delta f\left(n t_{s}-l T-\tau_{m, n}^{k}\right)} u\left(n t_{s}-q \Delta t-l T-\tau_{m, n}^{k}\right) e^{-j 2 \pi f_{c}\left(l T+\tau_{m, n}^{k}\right)}\right)_{M_{R} N \times 1} .
$$

The column vector $\mathbf{A}_{k}$ is the detecting signal corresponding to the $k$ th grid-cell. Then, we arrange $\left\{\mathbf{A}_{k}\right\}_{k=1}^{K}$ into an $M_{R} N \times K$ dimensional matrix $\mathbf{A}=\left(\begin{array}{llll}\mathbf{A}_{1} & \mathbf{A}_{2} & \cdots & \mathbf{A}_{K}\end{array}\right)$, which is often referred to as a dictionary matrix and defines the basis elements of the sparse representation.

Denote $\boldsymbol{\beta}=\left[\beta_{1}, \ldots, \beta_{K}\right]^{T} ; \boldsymbol{\beta}$ is an unknown vector to be recovered. Then, the measurement model reduces to (7), which is a familiar linear model used in most applications of sparse modeling:

$$
\mathbf{y}=\mathbf{A} \boldsymbol{\beta}+\mathbf{w}
$$

Hence, the estimation of scattering coefficients is reduced to recover the nonzero entries and the support set of the sparse vectors from the measurement vector $y$ and dictionary A.

2.2. Off-Grid RCI Model. The imaging equation presented in (7) is derived under the grid matching condition. However, in most cases of radar imaging, the scatterers are distributed in a continuous scene. Regardless of how finely the imaging scene is gridded, the scatterers may not lie at the grid-cell centers; then, the off-grid problem yields. Considering the off-grid, the actual scatterer location of the $k$ th scatterer can be expressed as

$$
\left(\bar{\theta}_{k}, \widehat{R}_{k}\right)=\left(\theta_{k}+\delta_{\theta k} \Delta_{\theta}, R_{k}+\delta_{R k} \Delta_{R}\right)
$$

where $\left\{\delta_{\theta k}, \delta_{R k}\right\} \in[-0.5,0.5]$ denotes the ratio between the position perturbation and grid size. Then, the measurement model in (7) could be concisely rewritten as $\mathbf{y}=\overline{\mathbf{A}} \boldsymbol{\beta}+\mathbf{w}$, where $\overline{\mathbf{A}}$ is the actual dictionary with respect to the actual scatterer location.

The OGE can be compensated by estimating the perturbation $\left\{\delta_{\theta k}, \delta_{R k}\right\}$. In other words, the off-grid RCI is a joint estimation problem to recover $\left\{\beta_{k}, \theta_{k}, \delta_{\theta k}, R_{k}, \delta_{R k}\right\}_{k=1}^{K}$ associated with the nonzero $\beta_{k}$. However, the estimation of $\left\{\delta_{\theta k}, \delta_{R k}\right\}$ is nonlinear optimization problem which is analytically intractable. Then, the first-order Taylor expansion is employed to approximate the model linearly:

$$
\begin{aligned}
& e^{-j 2 \pi\left(f_{c}+c_{m, q} \Delta f\right) \tau_{m, n}^{k}} \approx e^{-j 2 \pi\left(f_{c}+c_{m, q} \Delta f\right) \tau_{m, n}^{k}}(1 \\
& -j 2 \pi\left(f_{c}+c_{m, q} \Delta f\right) \\
& \left.\cdot\left(\left.\frac{\partial \tau_{m, n}^{k}}{\partial \theta}\right|_{\theta=\theta_{k}} \Delta_{\theta} \delta_{\theta k}+\left.\frac{\partial \tau_{m, n}^{k}}{\partial R}\right|_{R=R_{k}} \Delta_{R} \delta_{R k}\right)\right), \\
& \left.\frac{\partial \tau_{m, n}^{k}}{\partial \theta}\right|_{\theta=\theta_{k}}=\frac{1}{c}\left(\frac{(m-1) d_{T}}{d_{m, k}^{(T)}}+\frac{(n-1) d_{R}}{d_{n, k}^{(R)}}\right) R_{k} \cos \theta_{k}, \\
& \left.\frac{\partial \tau_{m, n}^{k}}{\partial R}\right|_{R=R_{k}}=\frac{1}{c}\left(\frac{R_{k}+(m-1) d_{T} \sin \theta_{k}}{d_{m, k}^{(T)}}\right. \\
& \left.+\frac{R_{k}+(n-1) d_{R} \sin \theta_{k}}{d_{n, k}^{(R)}}\right) .
\end{aligned}
$$

Define $\boldsymbol{\delta}_{\theta}=\left[\delta_{\theta 1}, \ldots, \delta_{\theta K}\right]^{T}$ and $\boldsymbol{\delta}_{R}=\left[\delta_{R 1}, \ldots, \delta_{R K}\right]^{T}$. Then, the actual dictionary $\overline{\mathbf{A}}$ could be approximated as a linear combination of $\left\{\boldsymbol{\delta}_{\theta}, \boldsymbol{\delta}_{R}\right\}$ :

$$
\overline{\mathbf{A}} \approx \mathbf{A}+\mathbf{A}_{\theta} \cdot \operatorname{diag}\left(\boldsymbol{\delta}_{\theta}\right)+\mathbf{A}_{R} \cdot \operatorname{diag}\left(\boldsymbol{\delta}_{R}\right),
$$

where $\mathbf{A}$ is the same matrix as in the grid matching scene and $\mathbf{A}_{\theta}$ and $\mathbf{A}_{R}$ are matrixes of size $M_{R} N \times K$ whose columns are

$$
\begin{aligned}
& \mathbf{A}_{\theta k} \operatorname{vec}\left\{\sum_{m=1}^{M_{T}} \sum_{l=0}^{L-1} \sum_{q=0}^{Q-1} e^{j 2 \pi\left(c_{m, q} \Delta f\left(n t_{s}-l T\right)-f_{c} l T\right)} e^{-j 2 \pi\left(f_{c}+c_{m, q} \Delta f\right) \tau_{m, n}^{k}}\right. \\
& \cdot u\left(n t_{s}-q \Delta t-l T-\tau_{m, n}^{k}\right) \cdot\left[-j 2 \pi\left(f_{c}+c_{m, q} \Delta f\right)\right. \\
& \left.\left.\left.\cdot \frac{\partial \tau_{m, n}^{k}}{\partial \theta}\right|_{\theta=\theta_{k}} \Delta_{\theta}\right]\right\}_{M_{R} N \times 1},
\end{aligned}
$$




$$
\begin{aligned}
& \mathbf{A}_{R k} \\
& =\operatorname{vec}\left\{\sum_{m=1}^{M_{T}} \sum_{l=0}^{L-1} \sum_{q=0}^{Q-1} e^{j 2 \pi\left(c_{m, q} \Delta f\left(n t_{s}-l T\right)-f_{c} l T\right)} e^{-j 2 \pi\left(f_{c}+c_{m, q} \Delta f\right) \tau_{m, n}^{k}}\right. \\
& \cdot u\left(n t_{s}-q \Delta t-l T-\tau_{m, n}^{k}\right) \cdot\left[-j 2 \pi\left(f_{c}+c_{m, q} \Delta f\right)\right. \\
& \left.\left.\left.\quad \cdot \frac{\partial \tau_{m, n}^{k}}{\partial R}\right|_{R=R_{k}} \Delta_{R}\right]\right\}_{M_{R} N \times 1} .
\end{aligned}
$$

Denote $\boldsymbol{\beta}_{\theta}=\boldsymbol{\delta}_{\theta} \odot \boldsymbol{\beta}$ and $\boldsymbol{\beta}_{R}=\boldsymbol{\delta}_{R} \odot \boldsymbol{\beta}$. Hence, the measurement model is also approximated by

$$
\mathbf{y} \approx \mathbf{A} \boldsymbol{\beta}+\mathbf{A}_{\theta} \boldsymbol{\beta}_{\theta}+\mathbf{A}_{R} \boldsymbol{\beta}_{R}+\mathbf{w}
$$

Obviously, $\boldsymbol{\beta}_{\theta}$ and $\boldsymbol{\beta}_{R}$ are also sparse vectors sharing the same support with $\beta$. Hence, (12) means that the imaging equation can be sparsely approximated using three known dictionaries $\left\{\mathbf{A}, \mathbf{A}_{\theta}, \mathbf{A}_{R}\right\}$. Now, the off-grid RCI becomes a joint sparse recovery problem to recover $\left\{\boldsymbol{\beta}, \boldsymbol{\beta}_{\theta}, \boldsymbol{\beta}_{R}\right\}$ jointly.

\section{Off-Grid Effects}

To reconstruct the target, some sparse recovery algorithms are commonly used. If the targets are located at the gridcell centers, the sparsity requirement is satisfied and sparse recovery works. However, the off-grid exists in general. In this section, the performance of the sparse-based estimation approaches is analyzed, by both mathematical analysis and numerical simulations.

3.1. The Off-Grid Effect on Imaging Quality. To investigate the off-grid, the measurement model in (12) is rewritten as

$$
\mathbf{y}=\mathbf{A} \boldsymbol{\beta}+\boldsymbol{\varepsilon}+\mathbf{w}
$$

where $\boldsymbol{\varepsilon} \approx\left(\mathbf{A}_{\theta} \cdot \operatorname{diag}\left(\boldsymbol{\delta}_{\theta}\right)+\mathbf{A}_{R} \cdot \operatorname{diag}\left(\boldsymbol{\delta}_{R}\right)\right) \boldsymbol{\beta}$ and denotes the gridding error caused by off-grid. As $\mathbf{A}$ is priorly known due to the prediscretized grids and the gridding error and noise are unknown, the reconstruction performance of the conventional sparse-based approaches would be depressed because the actual scattering-coefficient vector $\boldsymbol{\beta}$ is sparse in the actual dictionary $\overline{\mathbf{A}}$.

To illustrate the off-grid effect on the imaging quality, we make a quantitative analysis for the model in (13). The imaging quality discussed here is first indicated by the RIE, expressed as $\xi=20 \log _{10}\left(\|\overline{\boldsymbol{\beta}}-\boldsymbol{\beta}\|_{2} /\|\boldsymbol{\beta}\|_{2}\right)$ [3], where $\overline{\boldsymbol{\beta}}$ denotes the reconstructed scattering-coefficient vector. Then, the modeling error, composed of gridding error and external noise, is normalized as the SER, denoted as $\varsigma=$ $20 \log _{10}\left(\|\mathbf{y}\|_{2} /\left(\|\boldsymbol{\varepsilon}\|_{2}+\|\mathbf{w}\|_{2}\right)\right)$. Furthermore, the notion OGE is defined as $\zeta=\sum_{p=1}^{P}\left\|\boldsymbol{\delta}_{p}\right\|_{2} / P$ to measure the perturbations, where $\boldsymbol{\delta}_{p}=\left(\delta_{\theta p}, \delta_{R p}\right)^{T}$.

Next, an example is given to show how the OGE affects the imaging quality. Here, we only focus on the gridding error without external noise. The scattering-coefficient vector is calculated by BP algorithm. The radar system consists of eight transmitters and eight receivers with the interelement spacings $d_{T}=d_{R}=1 \mathrm{~m}$. The target space is $4 \mathrm{~m} \times 0.04 \mathrm{rad}$ and discretized to $20 \times 20$ grid-cells. The minimum $\mathrm{FH}$ interval and $\mathrm{FH}$ duration are $\Delta f=1 \mathrm{MHz}$ and $\Delta t=10 \mathrm{~ns}$, respectively. The length of FH codes $Q=400$ and $G=500$ and the maximum frequency is $G \Delta f=500 \mathrm{MHz}$. Further, four point scatterers are presented in Figure 2.

From the example, we could conclude that the sparse recovery performance and the imaging quality may degrade considerably in the presence of off-grid. As shown in Figure 2(f), the target image is badly blurred beyond recognition when $\zeta=0.0204$. The signal energy spills onto the offgrid components, and the energy spread renders the signal not sparse in the dictionary assumed for recovery. The target image in Figure 2(c) is recognizable when $\zeta=0.0136$, which requires $\varsigma \geq 1.656 \mathrm{~dB}$. It demonstrates that $\mathrm{BP}$ has low tolerance to the gridding error. A recognizable image with a small RIE requires a small gridding error or a high SER. However, as shown in Figure 3(a), the gridding error is seriously sensitive to the OGE, even when a small OGE can cause a large gridding error. The OGE more than 0.0204 even generates a negative SER, which means the gridding error almost overwhelms the received signal and creates an unfavorable condition for sparse recovery.

To realize the target reconstruction and improve the image quality, it is natural to consider a fine grid since the SER increases as the grids are refined [25]. However, two undesired effects emerge in this case: (a) the dictionary becomes increasingly coherent, rendering the sparse recovery ill-posed and (b) the larger size of the dictionary results in higher computational cost of reconstruction.

Consequently, the question is presented: "is it possible to adapt to the highly coherent dictionary based on a finer grid to combat the off-grid, while achieving a robust sparse reconstruction?" To answer the question, the sparse recovery, based on the band exclusion (BE) and local optimization (LO), is discussed in the next section.

3.2. The CCRB under Off-Grid. To investigate the off-grid effect deeply, the sparse recovery could be reformulated as a parameter estimation problem. The sparse vector $\beta$ is viewed as a deterministic parameter vector, and $\mathbf{y}$ represents the observation data. The metric for the recovery performance is mean square error (MSE), that is, $E\left\{\|\boldsymbol{\beta}-\overline{\boldsymbol{\beta}}\|_{2}^{2}\right\}$, which is a good way to capture the system performance and commonly used in radar application. For MSE, its lower bound (i.e., CRB) has been well established for parameter vector without further constraints. Recently, researches on the lower bound of MSE for constrained parameter vectors (i.e., CCRB), especially sparse parameter vectors, have been developed $[26,27]$. Hence, we focus on the theoretical CCRB of sparse estimators in the presence of off-grid. However, the CCRB is complex to calculate directly. Fortunately, in the case of sparse estimation under Gaussian noise, the CCRB is identical to the MSE of the "oracle" estimator, which is defined as the least squares solution within the true support set [28]; that is, 


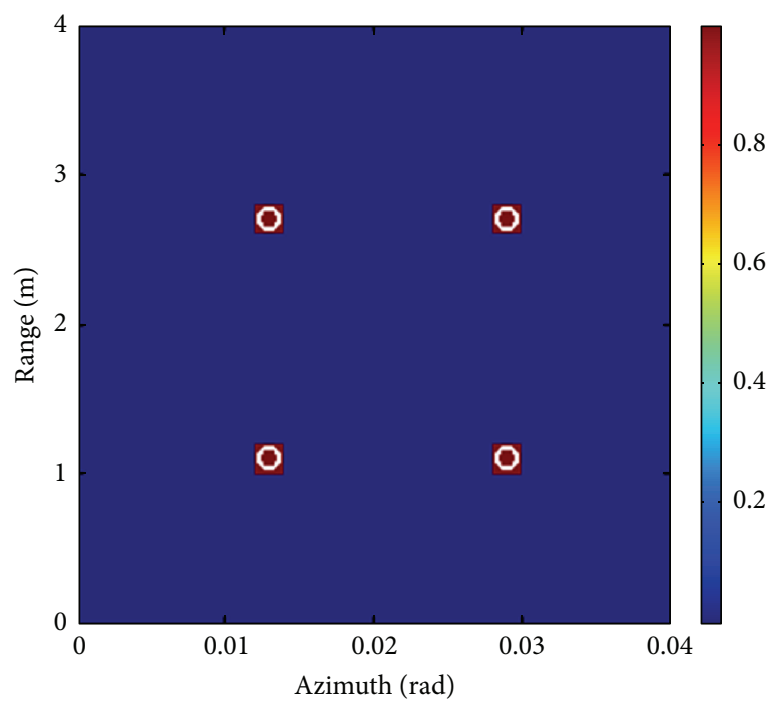

(a) $\zeta=0$

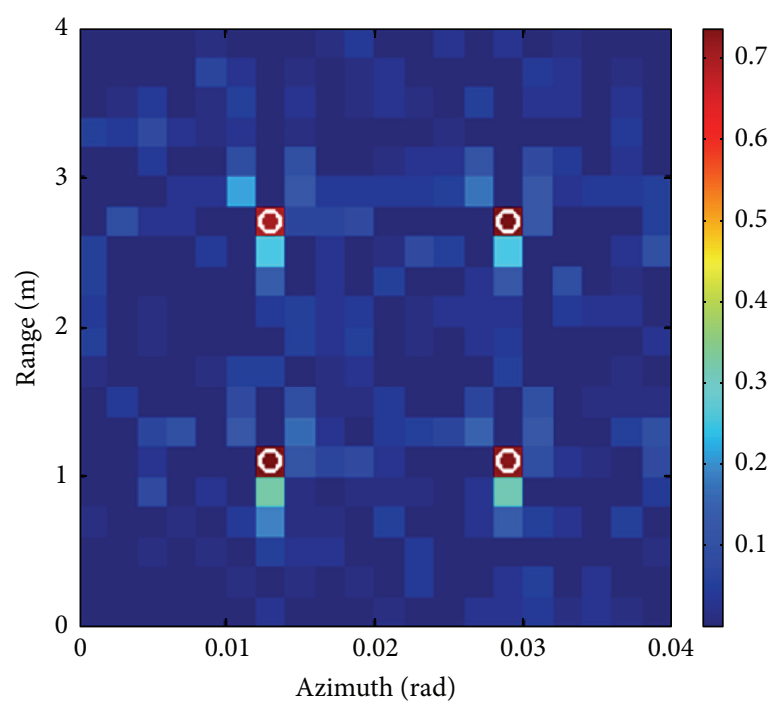

(c) $\zeta=0.0136$

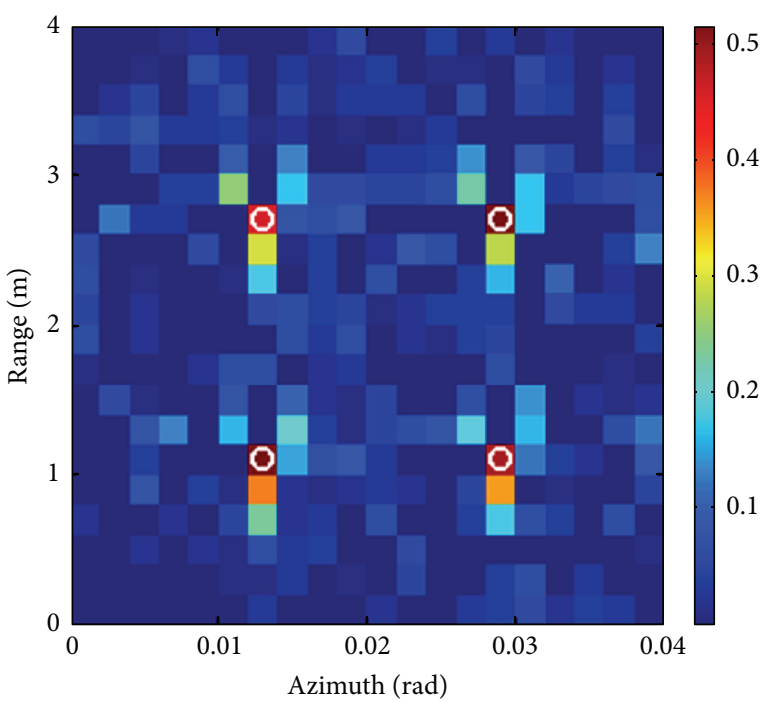

(e) $\zeta=0.0181$

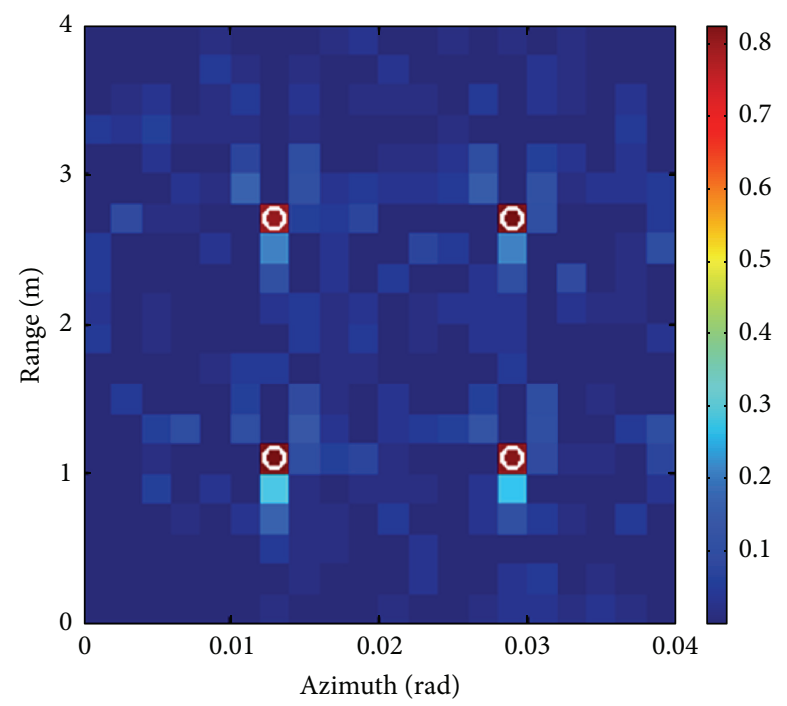

(b) $\zeta=0.0113$

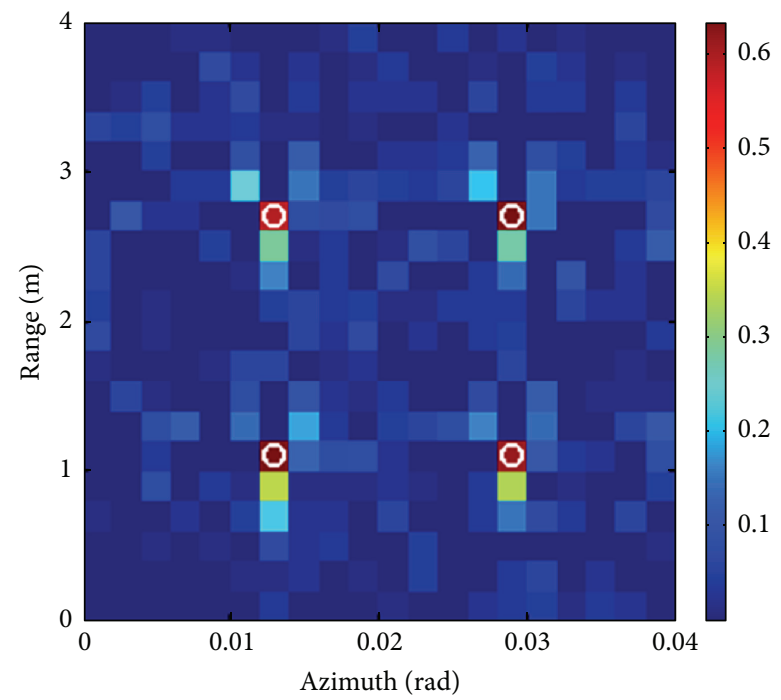

(d) $\zeta=0.0158$

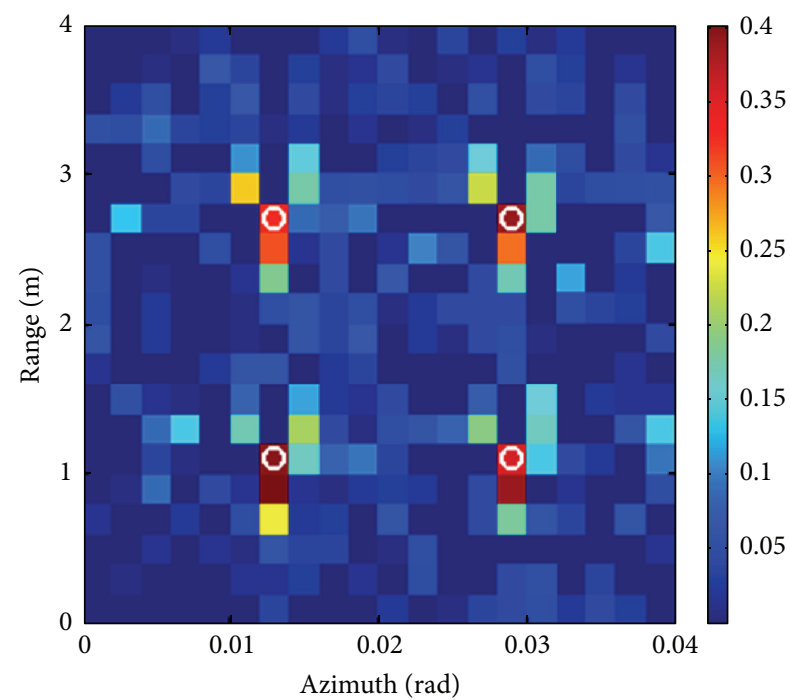

(f) $\zeta=0.0204$

FIGURE 2: RCI results when $\zeta=\{0,0.0113,0.0136,0.0158,0.0181,0.0204\}$. 


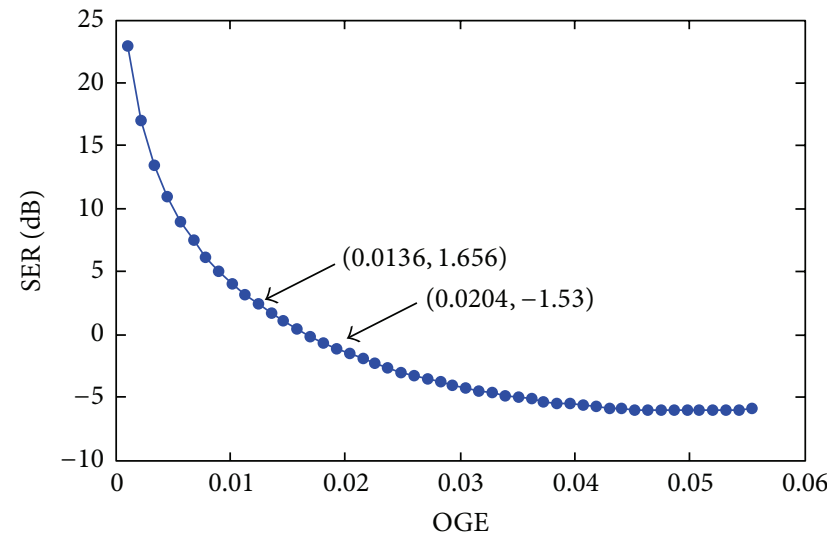

(a)

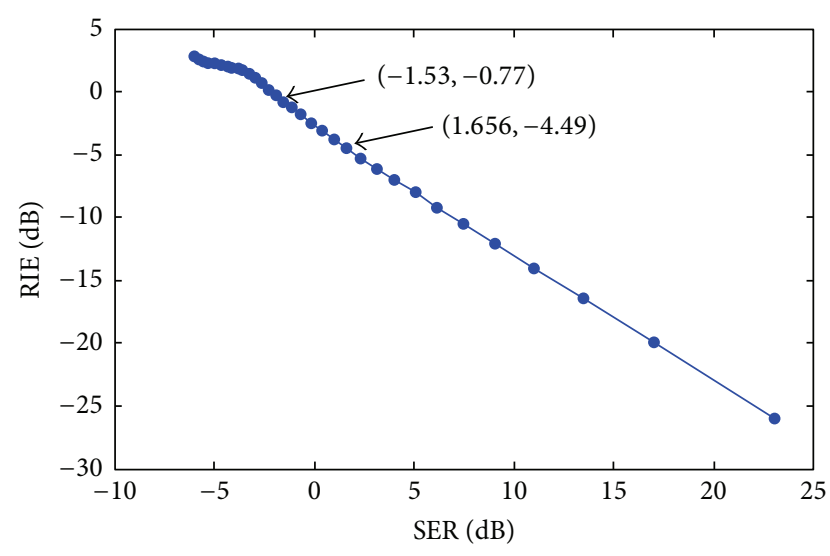

(b)

FIGURE 3: Off-grid effects on imaging quality. (a) SER versus OGE. (b) RIE versus SER.

$\overline{\boldsymbol{\beta}}_{\text {or }}=\arg \min _{\operatorname{supp}(\tilde{\boldsymbol{\beta}}) \subseteq S}\|\boldsymbol{\beta}-\tilde{\boldsymbol{\beta}}\|_{2}^{2}$. Thus, the goal is changed to achieve the MSE of the oracle estimator in the presence of offgrid. Using the notation introduced above, we have

$$
\begin{aligned}
& \left(\overline{\boldsymbol{\beta}}_{\mathrm{or}}\right)_{S}=\mathbf{A}_{S}^{\dagger} \mathbf{y}, \\
& \left(\overline{\boldsymbol{\beta}}_{\mathrm{or}}\right)_{S^{C}}=\mathbf{0},
\end{aligned}
$$

where $\mathbf{A}_{S}$ is the dictionary associated with the support set $S$ and $S^{C}$ is the complement of $S$. The oracle estimator is not a true estimator as $\overline{\boldsymbol{\beta}}_{\text {or }}$ relies on the knowledge of $S$ which is unknown generally.

Considering the off-grid, the actual measurement model is shown in (13), and then the reconstructed result is $(\boldsymbol{\beta})_{S}=$ $\mathbf{A}_{S}^{\dagger}(\mathbf{y}-\boldsymbol{\varepsilon})$ theoretically. However, in the mathematical model of sparse reconstruction procedure, the echo signal is composed as $\mathbf{y}=\mathbf{A} \overline{\boldsymbol{\beta}}+\mathbf{w}$. Thus, we could obtain the result of oracle estimator $(\overline{\boldsymbol{\beta}})_{S}=\mathbf{A}_{S}^{\dagger} \mathbf{y}$, as described in (14). Then, the MSE of the oracle estimator (i.e., CCRB) in the presence of off-grid can be computed directly

$$
\operatorname{CCRB}(\boldsymbol{\beta})=\operatorname{MSE}(\overline{\boldsymbol{\beta}}-\boldsymbol{\beta})=\|\overline{\boldsymbol{\beta}}-\boldsymbol{\beta}\|_{2}^{2}=\left\|\mathbf{A}_{S}^{\dagger} \boldsymbol{\varepsilon}\right\|_{2}^{2} .
$$

Therefore, we can conclude from (15) that the CCRB is determined by both the dictionary corresponding to the support set and the gridding error. Notice that the gridding error is defined as $\boldsymbol{\varepsilon} \approx\left(\mathbf{A}_{\theta} \cdot \operatorname{diag}\left(\boldsymbol{\delta}_{\theta}\right)+\mathbf{A}_{R} \cdot \operatorname{diag}\left(\boldsymbol{\delta}_{R}\right)\right) \boldsymbol{\beta}$; then, the OGE would affect the CCRB ultimately. To show this effect directly, we give a numerical example and show that the $\mathrm{CCRB}$ is roughly proportional to the OGE, which is shown in Figure 4.

\section{Image Reconstruction Approach}

Since the SER is roughly inverse proportion to the grid size, it is possible to improve the sparse recovery performance by grid refinement. However, it is still not an advisable approach, as a denser grid may lead to higher computational cost and dramatically enhance the mutual coherence among the nearby columns of the dictionary which causes the violation of restricted isometry property (RIP) condition for reliable sparse recovery. In this section, the BLOOMP algorithm [25], which embeds BE and LO into OMP, is extended to $2 \mathrm{D}$ version and then applied to off-grid RCI. This modified algorithm alleviates the off-grid effect and realizes the grid matching which means that the off-grid scatterers are captured by the closest grid-cell centers. Next, following the grid matching stage, the OGE is calibrated precisely by the AIM algorithm.

4.1. Grid Matching Based on 2D BLOOMP. To ensure the sparse reconstruction performance, all the columns within A should be orthogonal. First, define the coherence measure of the dictionary $\mathbf{A}$ as $\mu(\mathbf{A})=\max _{k \neq 1} \mu(k, l)$, where $\mu(k, l)=$ $\left|\left\langle\mathbf{A}_{k}, \mathbf{A}_{l}\right\rangle\right| /\left|\mathbf{A}_{k}\right|\left|\mathbf{A}_{l}\right|$, and $\mathbf{A}_{k}$ and $\mathbf{A}_{l}$ denote the columns of $\mathbf{A}$. If two columns are highly coherent, that is, $\mu(\mathbf{A}) \approx 1$, it is nearly impossible to distinguish whether the signal comes from one or the other.

As shown in Figure 2, the off-grid scatterer will spill nonzero values into all grid-cells. Besides, in the case of a denser grid, the increasing coherence of dictionary makes it hard to ensure the sparse reconstruction. To alleviate the problem, the $\mathrm{BE}$ is introduced.

Firstly, define the notion coherence band as [25]

$$
\begin{aligned}
& B_{\eta}(k)=\{i \mid \mu(i, k)>\eta\}, \\
& B_{\eta}(S)=\bigcup_{k \in S} B_{\eta}(k),
\end{aligned}
$$

where $B_{\eta}(k)$ and $B_{\eta}(S)$ are the set of the $\eta$-coherence band of the index $k$ and set $S$, respectively. Due to the symmetry 


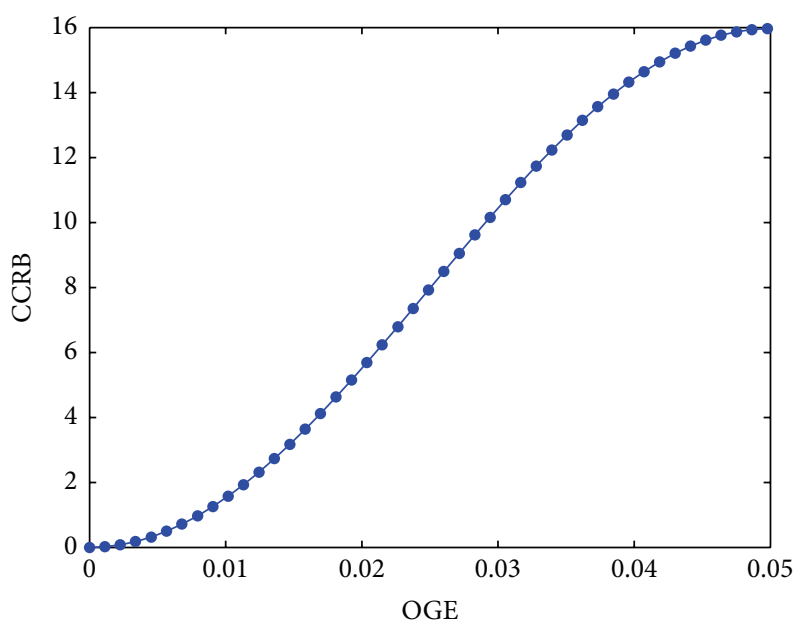

Figure 4: MSE increases with the OGE.

$\mu(i, k)=\mu(k, i), \forall i, k, i \in B_{\eta}(k)$ if and only if $k \in B_{\eta}(i)$. Denote the secondary coherence band as

$$
\begin{aligned}
& B_{\eta}^{(2)}(k) \equiv B_{\eta}\left(B_{\eta}(k)\right)=\bigcup_{j \in B_{\eta}(k)} B_{\eta}(j), \\
& B_{\eta}^{(2)}(S) \equiv B_{\eta}\left(B_{\eta}(S)\right)=\bigcup_{k \in S} B_{\eta}^{(2)}(k) .
\end{aligned}
$$

If the scatterers are beyond each other's coherence band, it is possible to localize the scatterers within their respective coherence bands, no matter how large the mutual coherence is. If the scatterers are sufficiently separated with respect to the coherence band, the support can be approximately reconstructed. BE takes advantage of the prior information of widely separated scatterers and can be easily embedded in the greedy algorithm, that is, OMP.

To embed BE into OMP, the matching step is changed as

$$
\begin{aligned}
& i_{\text {max }}=\underset{i}{\arg \min }\left|\left\langle r^{n-1}, \mathbf{A}_{i}\right\rangle\right|, \\
& i \in\left\{k=u_{(k)} U+v_{(k)} \mid u_{(k)} \notin B_{\eta_{\theta}}^{(2)}\left(S_{\theta}^{n-1}\right), v_{(k)} \notin B_{\eta_{R}}^{(2)}\left(S_{R}^{n-1}\right)\right\},
\end{aligned}
$$

where $\left(u_{(k)}, v_{(k)}\right)$ represents the $k$ th grid in the discretized scene. $\eta_{\theta}$ and $\eta_{R}$ are the mutual coherence thresholds for azimuth and range, respectively. $B_{\eta}^{(2)}\left(S_{\theta}^{n-1}\right)=\bigcup_{k \in S_{\theta}^{n-1}} B_{\eta_{\theta}}^{(2)}(k)$, where $S_{\theta}^{n-1}$ denotes the azimuth support set in the previous $(n-1)$ th iteration and $B_{\eta_{\theta}}^{(2)}\left(S_{R}^{n-1}\right)$ is defined likewise. Thus, in the current search, the double $\eta$-band of the estimated support in the previous iteration is avoided on the azimuth and range spaces synchronously.

If the elements of the support set are sufficiently separated and the dynamic range is small, the demand for the incoherence of dictionary is degraded, and the regular grid could be denser. Thus, the off-grid effect is alleviated to a certain extent. Since dynamic range of scatterers is an essential factor determining the performance of recovery, the sensitivity to dynamic range can be drastically reduced by the LO approach.

LO is a residual-reduction technique applied to the current support set $S^{k}$, where the residual $\|\mathbf{A} \overline{\boldsymbol{\beta}}-\mathbf{y}\|_{2}$ is minimized by varying one location at one time while all other locations are fixed. In each step, the support of $\bar{\beta}$ differs from $S^{n}$ by at most one index in the coherence band of $S^{n}$, but its amplitude is chosen to minimize the residual by solving the least squares problem.

For the LO step, the search is also operated on the azimuth dimension and range dimension, respectively. In the $n$th iteration, the support is updated as

$$
\begin{aligned}
& S^{n}=\operatorname{supp}\left(\boldsymbol{\beta}^{n}\right), \quad \text { where } \boldsymbol{\beta}^{n}=\underset{\overline{\boldsymbol{\beta}}}{\arg \min }\|\mathbf{A} \overline{\boldsymbol{\beta}}-\mathbf{y}\|_{2} \\
& \text { s.t. } \quad \operatorname{supp}(\overline{\boldsymbol{\beta}}) \\
& \quad=\left\{k=u_{(k)} U+v_{(k)} \mid u_{(k)}=\left(S_{\theta}^{n-1} \backslash\left\{i_{n}\right\}\right) \cup\left\{j_{n}\right\}, j_{n} \in B_{\eta_{\theta}}\left(\left\{i_{n}\right\}\right), v_{(k)}=\left(S_{R}^{n-1} \backslash\left\{i_{n}\right\}\right) \cup\left\{j_{n}\right\}, j_{n} \in B_{\eta_{R}}\left(\left\{i_{n}\right\}\right)\right\} .
\end{aligned}
$$

Finally, the 2D BLOOMP algorithm, which realizes the grid matching by imbedding $\mathrm{BE}$ and LO into OMP, is described in Algorithm 1.

For the 2D BLOOMP, the two critical parameters, that is, the radius of excluded band in $\mathrm{BE}$ and radius of searching band in LO, should be confirmed first.

As assumed above, the scatterers should be well separated more than the resolution limit (RL), to confirm that the target image is well reconstructed. The $\mathrm{RL}$ is closely related to the decay property of the mutual coherence $\mu(\mathbf{A})$ in the sparse setting. Accordingly, the coherence band can be obtained after $\mu(\mathbf{A})$ is calculated. The radius of excluded band should be larger than the coherence band. The spacing among the scatterers should be more than two times the radius of excluded band, or the scatterers would be badly separated. As the local search grid varies through the resolution cell, the radius of LO should be also larger than the coherence band. 


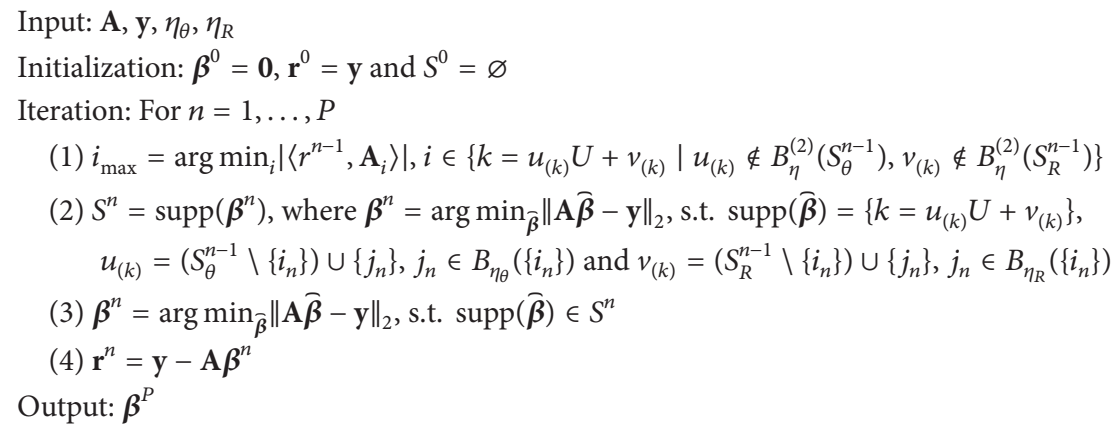

Algorithm 1: 2D BLOOMP algorithm.

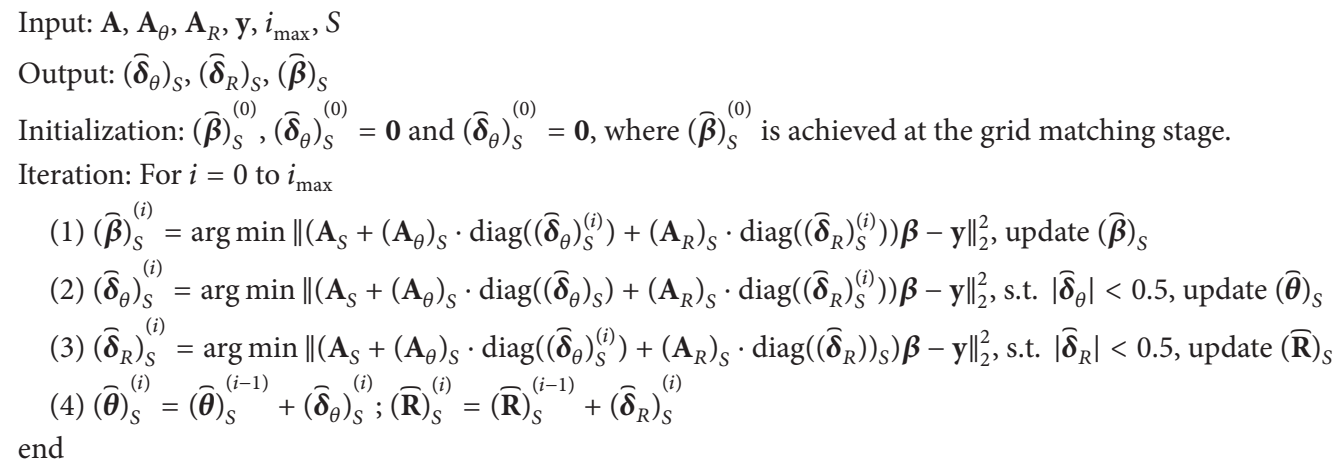

Algorithm 2: AIM algorithm.

Even if the performance of $2 \mathrm{D}$ BLOOMP is improved significantly, the performance guarantee is hard to prove theoretically. In general, 2D BLOOMP enhances the success probability of recovery and is robust to noise and dynamic range.

4.2. OGE Calibration. The 2D BLOOMP algorithm presented as Algorithm 1 could alleviate the off-grid and realize the grid matching in the case of a denser grid. The rough image of the target is achieved and associated with the actual image in the absence of off-grid. However, the reconstructed image is not the same as the actual image when off-grid exists, as the location perturbation $\left\{\delta_{\theta k}, \delta_{R k}\right\}$ is not calibrated. Therefore, the OGE calibration should be executed.

In this part, the off-grid is compensated by creating the dictionary iteratively, with both azimuth and range varying at each iteration. This allows us to create the dictionary with varying mismatch iteratively using previous computed dictionary.

Remark. When $\beta_{k}=0$ for certain $k, \delta_{\theta k}$ and $\delta_{R k}$ can take any value without any contributions to the observation vector $\mathbf{y}$, so recovering such $\delta_{\theta k}$ and $\delta_{R k}$ is unnecessary. Thus, only the entries of $\delta_{\theta k}$ and $\delta_{R k}$ related to nonzero entries of $\boldsymbol{\beta}$, denoted as $\left(\boldsymbol{\delta}_{\theta}\right)_{S}$ and $\left(\boldsymbol{\delta}_{R}\right)_{S}$, are recovered, respectively.

In this stage, for the grids which are achieved by $2 \mathrm{D}$ BLOOMP and close to the scatterers, alternating iterations are run to find the actual scatterers within the corresponding grid-cells. Here, the idea is to find $\left(\boldsymbol{\delta}_{\theta}, \boldsymbol{\delta}_{R}\right)$ that minimizes the residual $\|\overline{\mathbf{A}} \overline{\boldsymbol{\beta}}-\mathbf{y}\|_{2}$. This is achieved by iteratively calculating the dictionary at the current location, starting from the current grid-cell center, and estimating $\left(\left(\boldsymbol{\delta}_{\theta}\right)_{S},\left(\boldsymbol{\delta}_{R}\right)_{S}\right)$. The iterations can be terminated if the residual is smaller than a predetermined threshold. In the proposed method, the model data corresponding to the final locations are generated, and the residual is calculated by projecting the measurements to the span of model data. This procedure provides a better fit to the measurements by decreasing the gridding error.

Notice. To reduce computation complexity, the range and azimuth locations are estimated separately. Furthermore, the scattering-coefficient vector $\boldsymbol{\beta}$ is also updated at each iteration.

Consequently, the OGE calibration method, called AIM algorithm, is proposed in Algorithm 2.

\section{Discussion and Numerical Analysis}

In this section, the performance of the proposed method is analyzed for off-grid FH-RCI.

An X-band RCI radar system with carrier frequency of $10 \mathrm{GHz}$ is considered. Both the transmitters and receivers are configured as ULAs with the numbers of elements $M_{T}=$ $M_{R}=8$ and interelement spacings $d_{T}=d_{R}=1 \mathrm{~m}$. The 


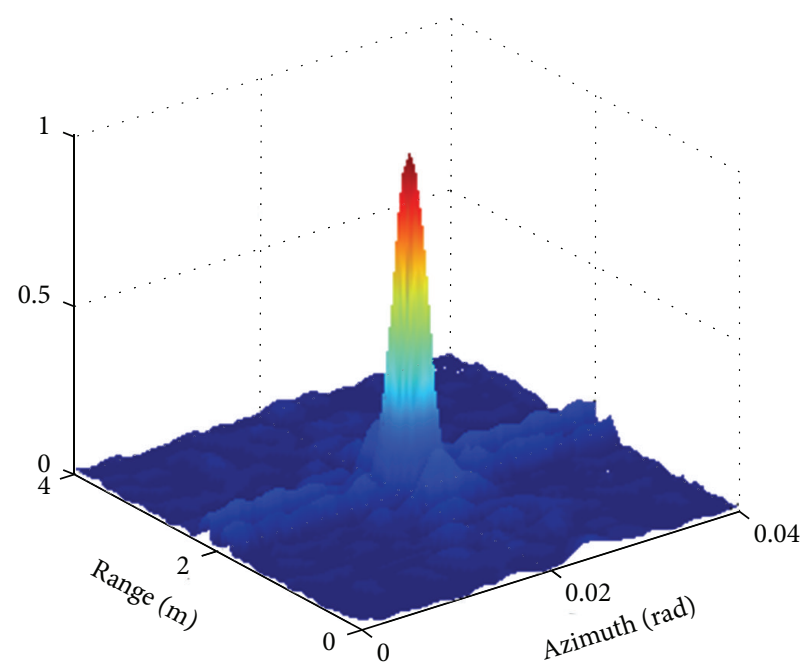

(a)

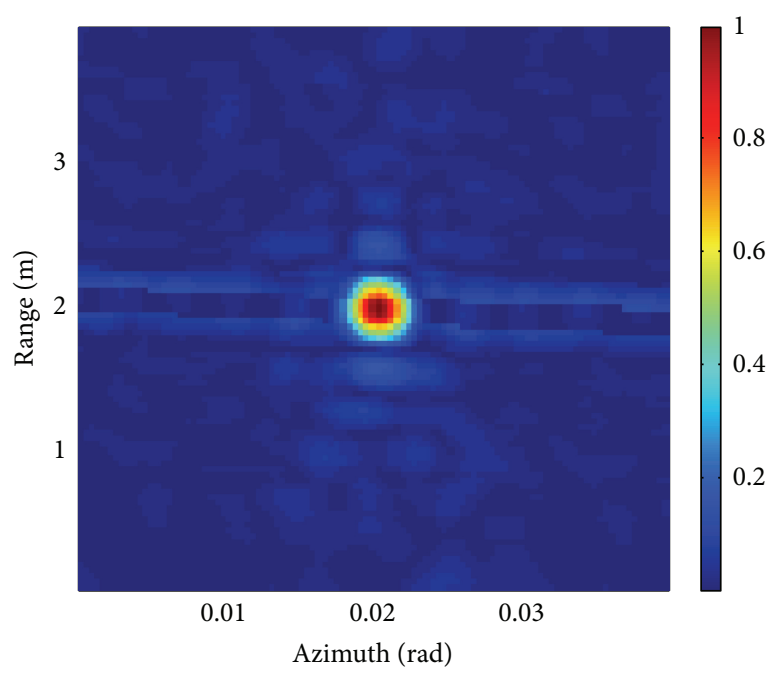

(b)

FIgURE 5: The PSF of point (0.02 rad, $2 \mathrm{~m}$ ); (b) is the top view of (a).

minimum $\mathrm{FH}$ interval is $\Delta f=1 \mathrm{MHz}$ and the maximum frequency is $500 \mathrm{MHz}$. And the waveforms are comprised by $Q=400$ hopping frequencies with the $\mathrm{FH}$ duration $\Delta t=10 \mathrm{~ns}$. A $2 \mathrm{D}$ range-azimuth target space, covering $4 \mathrm{~m} \times$ $0.04 \mathrm{rad}$, is discretized to $20 \times 20$ grid-cells, each of which is $0.2 \mathrm{~m} \times 0.002 \mathrm{rad}$. Further, there are supposed to be four widely separated point scatterers which are randomly located within the grid-cells.

5.1. Verification of $2 D B L O O M P$. In this part, we verify the performance of $2 \mathrm{D}$ BLOOMP by numerical simulations. Besides 2D BLOOMP, other typical algorithms will be involved, that is, Matched Filter (MF), OMP, Basis Pursuit Denoising (BPDN), S-TLS [20], and CP [3].

At first, the OGE is assumed to be $\zeta=0.2546$ and the external noise is fixed at SNR $=20 \mathrm{~dB}$. The coarse grid is refined by 5 times. Before the numerical experiments, the radii of $\mathrm{BE}$ and $\mathrm{LO}$ should be confirmed first. As the analytical expression of $\mu(\mathbf{A})$ is difficult to calculate, the point spread function (PSF) is simulated to investigate the pairwise coherence between one grid-cell center and others. As shown in Figure 5, the PSF of location (0.02 rad, $2 \mathrm{~m}$ ) presents a marked peak and the side lobe could be ignored; thus, the resolution performance of $\mathrm{FH}$ waveforms is excellent comparing with other waveforms, that is, LFM waveforms. According to the main lobe of PSF in Figure 5(b), the resolution cell is about $0.6 \mathrm{~m} \times 0.0064 \mathrm{rad}$. The radii of excluded band in $\mathrm{BE}$ and searching band in LO could be set up as $r_{1}=8$ and $r_{2}=10$, respectively, according to the aforementioned method.

Figure 6 shows the target images reconstructed by the six algorithms, where the white circles represent the actual location of scatterers. As presented in the figure, the target images by MF and BPDN nearly fail and the energy spills over the target space, because of the off-grid. Comparing with Figures 6(a) and 6(c), the image quality shown in Figures 6(b), 6(d), and 6(e) is improved significantly, mainly as the prior knowledge of sparsity is utilized and the corresponding algorithms are stable to off-grid to some extent. However, there are several "illusive" scatterers around the true scatterers, which will lead to failure in picking up the scatterers without other prior knowledge (i.e., the number and scattering coefficient of scatterers). In contrast, for Figure 6(f), the image quality is the best among Figure 6, the scatterers are located more precisely, and the "illusive" scatterers are not quite significant. Consequently, comparing with other algorithms tested above, 2D BLOOMP shows the best performance.

5.2. Performance Experiments of $2 D$ BLOOMP. In this subsection, the performance of 2D BLOOMP is investigated by numerical experiments and comparing with other algorithms. The performance is evaluated by RIE with respect to SNR and OGE, as they are clearly essential factors determining the reconstruction performance.

Figure 7(a) shows the RIE with respect to the SNR varying from $0 \mathrm{~dB}$ to $40 \mathrm{~dB}$. The RIE is averaged over 50 trials. In each trial, the OGE is fixed at a small value $\zeta=1.4 \times 10^{-4}$; thus, the SER is dominated mainly by the external noise, not the gridding error.

As shown in Figure $7(\mathrm{a}), \mathrm{MF}$ is robust to noise for the RIE remains nearly unchanged, while other algorithms are sensitive to noise and the image quality is improved significantly as SNR increases. 2D BLOOMP performs the best among the listed algorithms when SNR is smaller than $16 \mathrm{~dB}$. However, the RIE does not decrease for SNR of over $16 \mathrm{~dB}$, while other algorithms, especially S-TLS and BPDN, continue to improve the quality because they can cope with perturbations present in both the received signal and the dictionary. As there exists external noise, the RIE could not be arbitrarily low.

Then, it seems that the presented 2D BLOOMP does not perform much better than other algorithms. However, the algorithm is operated in the refinement grid, which 


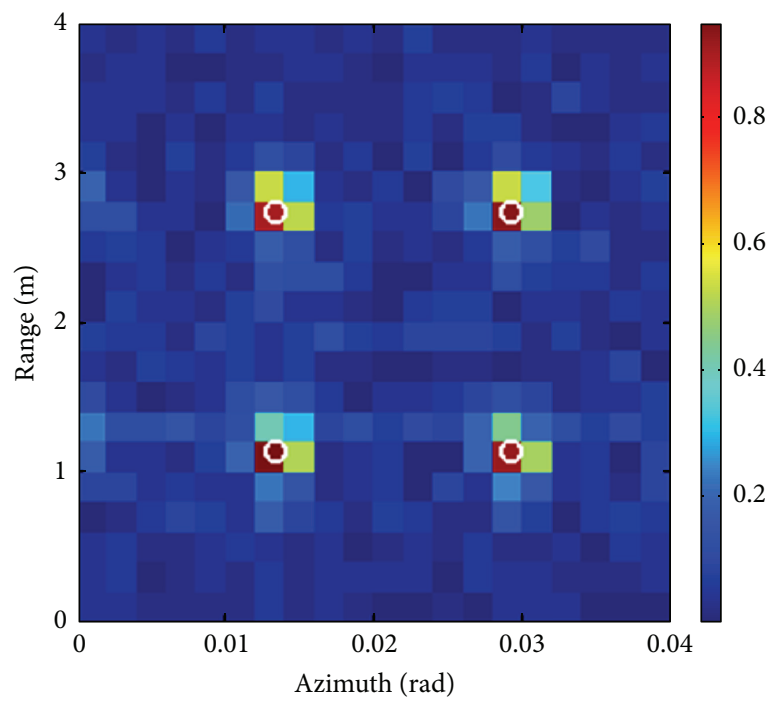

(a)

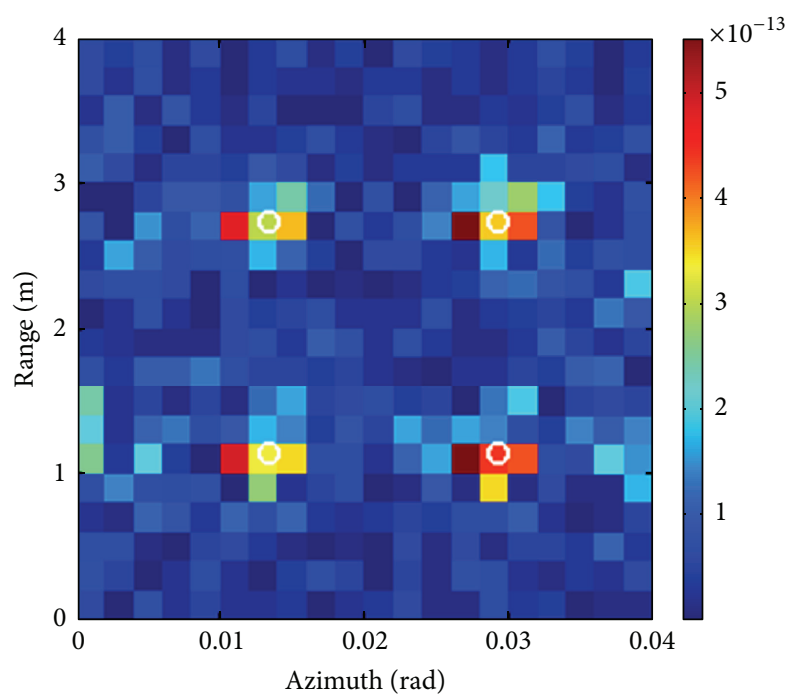

(c)

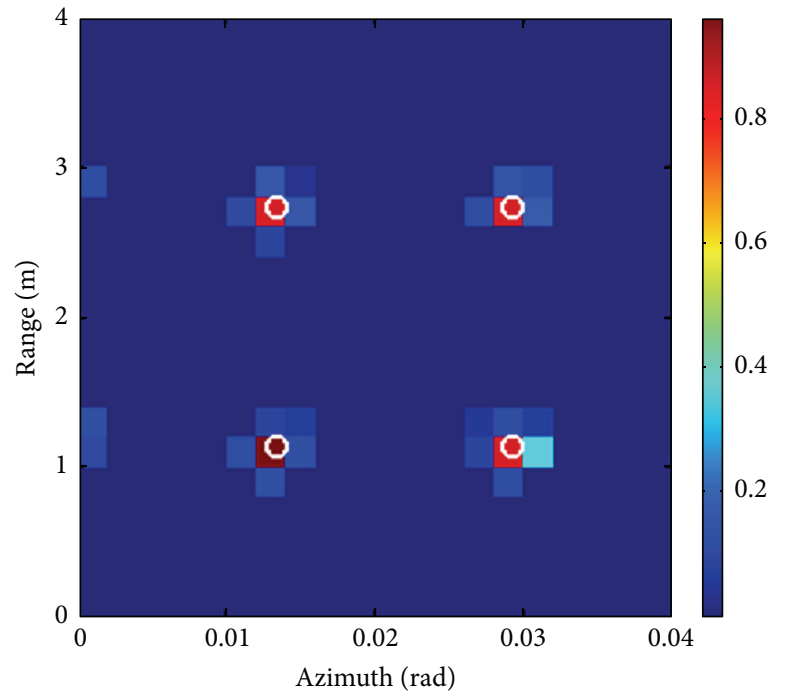

(e)

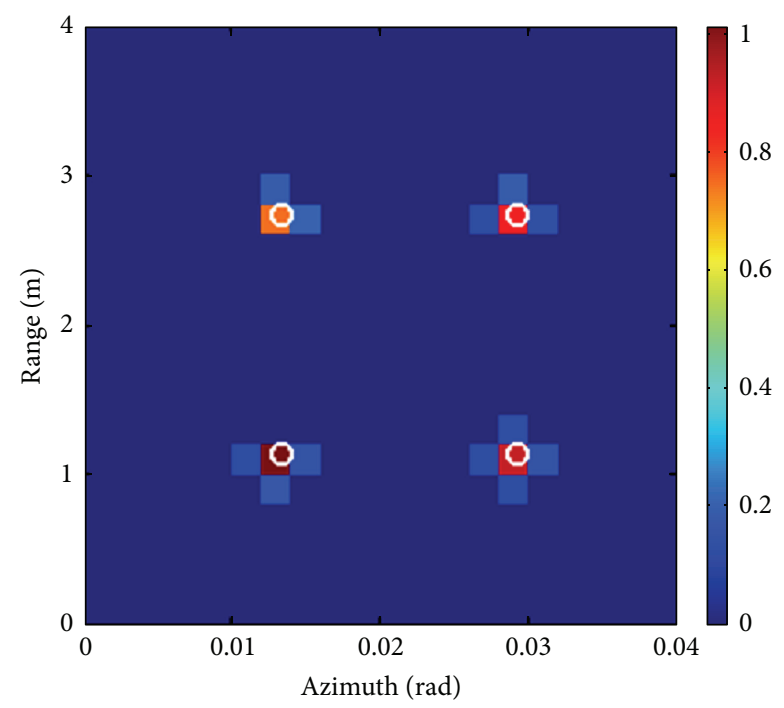

(b)

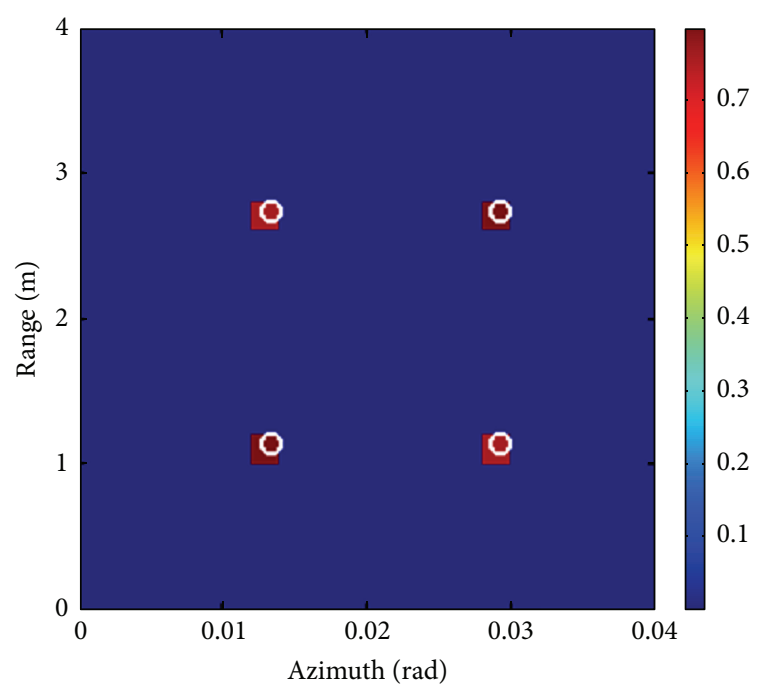

(d)

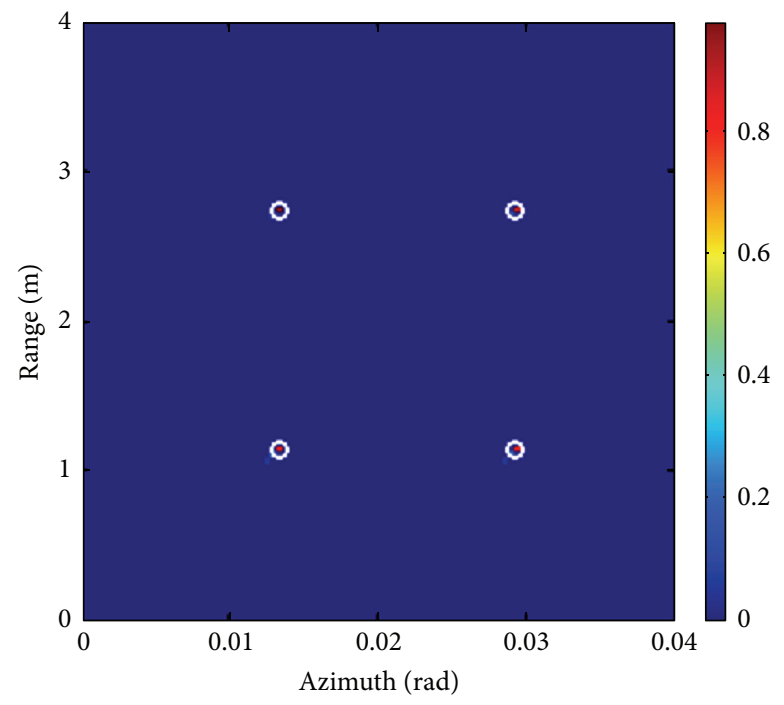

(f)

Figure 6: Off-grid RCI results. (a) MF, (b) OMP, (c) BPDN, (d) S-TLS, (e) CP, and (f) 2D BLOOMP. 


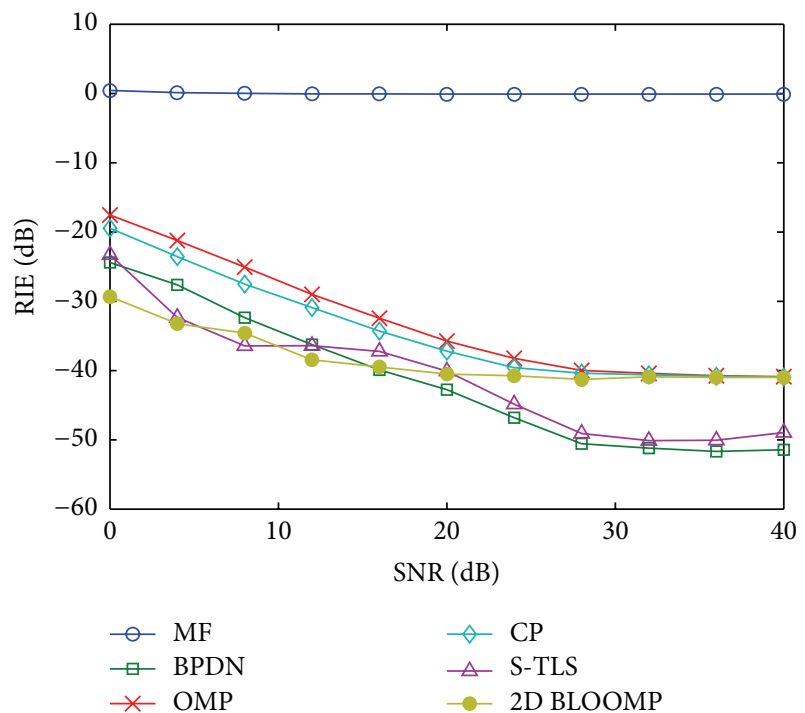

(a)

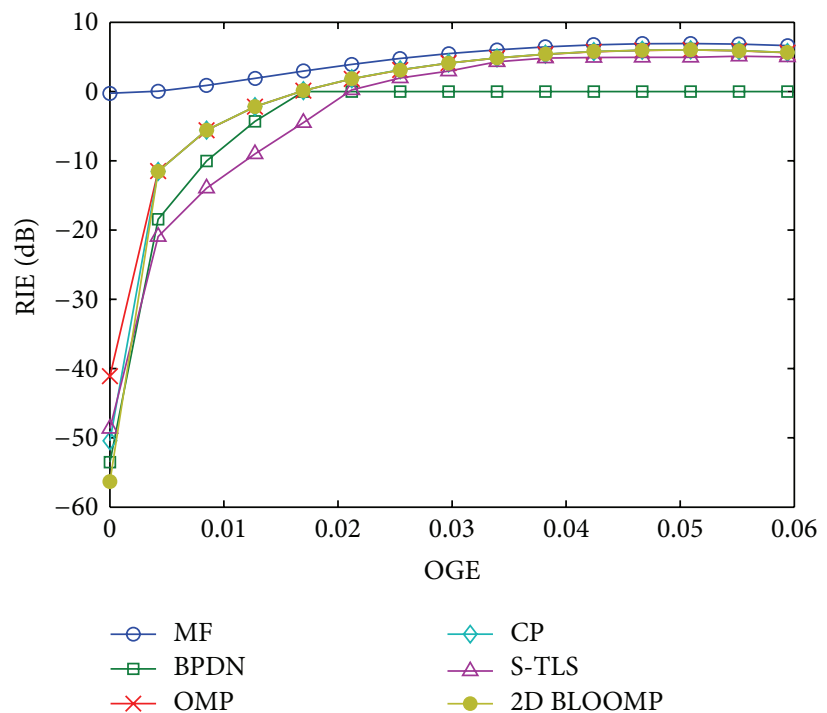

(b)

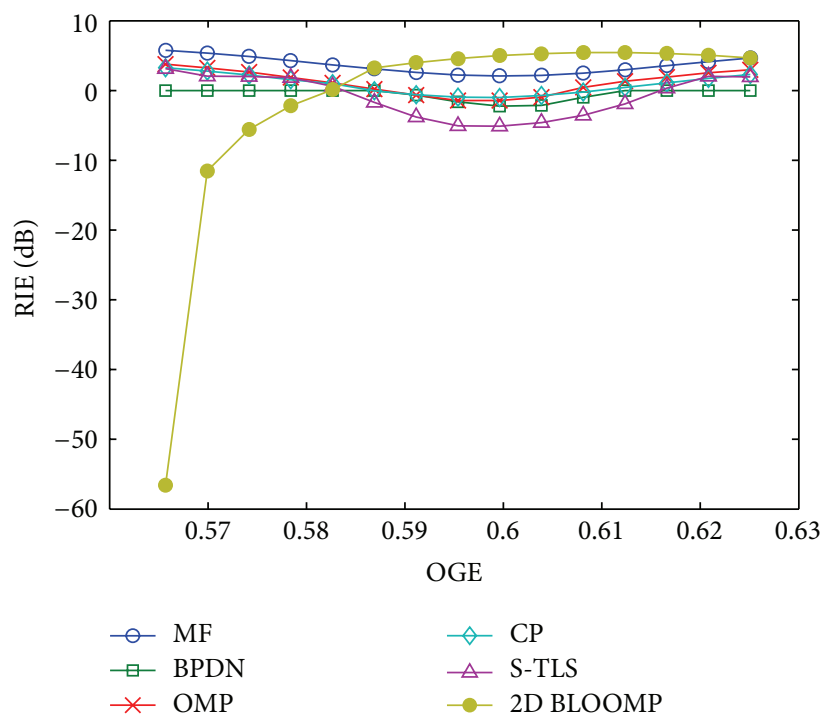

(c)

FIGURE 7: Results of performance experiments. (a) RIE versus SNR in the presence of off-grid. (b) RIE versus OGE ( $\zeta=0 \sim 0.059$ ). (c) RIE versus OGE $(\zeta=0.566 \sim 0.625)$.

still performs well and localizes the scatterers precisely. While for OMP, BPDN, S-TLS, and CP, the performance will degrade rapidly when the target scene is refined and the OGE increases, as shown in Figure 8.

Another performance criterion is the RIE under different OGEs. Figures 7(b) and 7(c) present the corresponding results where the RIE increases rapidly with OGE, which means that the image quality is worse. When OGE is larger than 0.02 , RIE is over $0 \mathrm{~dB}$ indicating that the imaging error $\|\overline{\boldsymbol{\beta}}-\boldsymbol{\beta}\|_{2}$ almost overwhelms $\|\boldsymbol{\beta}\|_{2}$; thus, the off-grid effect emerges. Interestingly, the RIE remains unchanged at $0 \mathrm{~dB}$ for BPDN; the reason is expressed by Figure 6(c): the energy spills all over the target space and the amplitudes are slight. The phenomenon can explain the off-grid effect to some extent. 2D BLOOMP, whose performance is the same as OMP, does not show the advantage over other algorithms except MF, because the OGE is small and the scatterers are distributed within the refined grid-cells. However, when the OGE increases beyond the refined grid-cells, the advantage of 2D BLOOMP emerges, as shown in Figure 7(c). For other algorithms, the RIEs maintain a high level when the OGE increases, while the RIE for 2D BLOOMP becomes small. The reason is that the OGE increases and the scatterers are located within the coarse grid-cells; the gridding error also increases. However, 2D BLOOMP is operated in the refinement grid during the increasing process; the scatterers may be beyond the refined grid-cells but captured by other grid-cells. Furthermore, the SER increases after the grids are refined. 


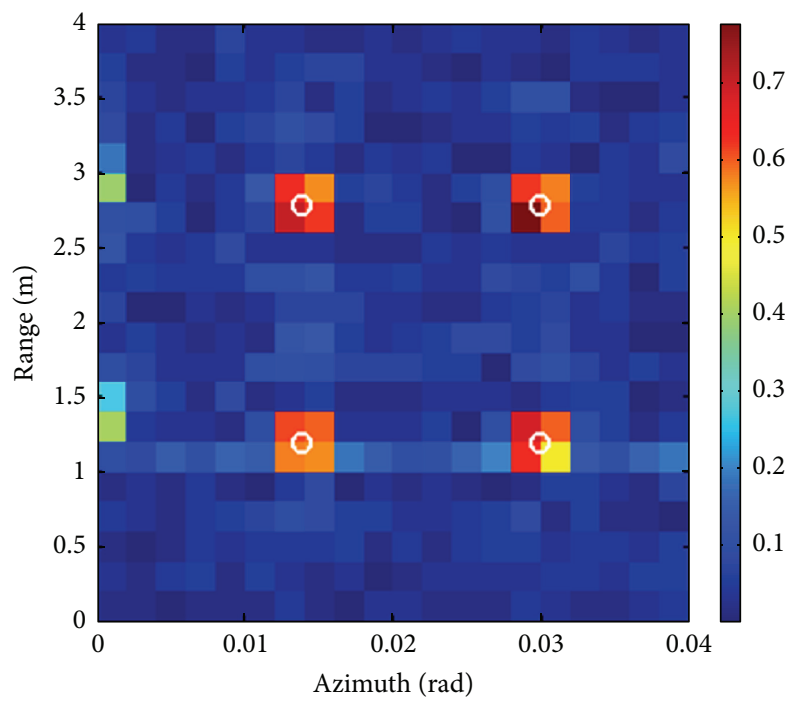

(a)

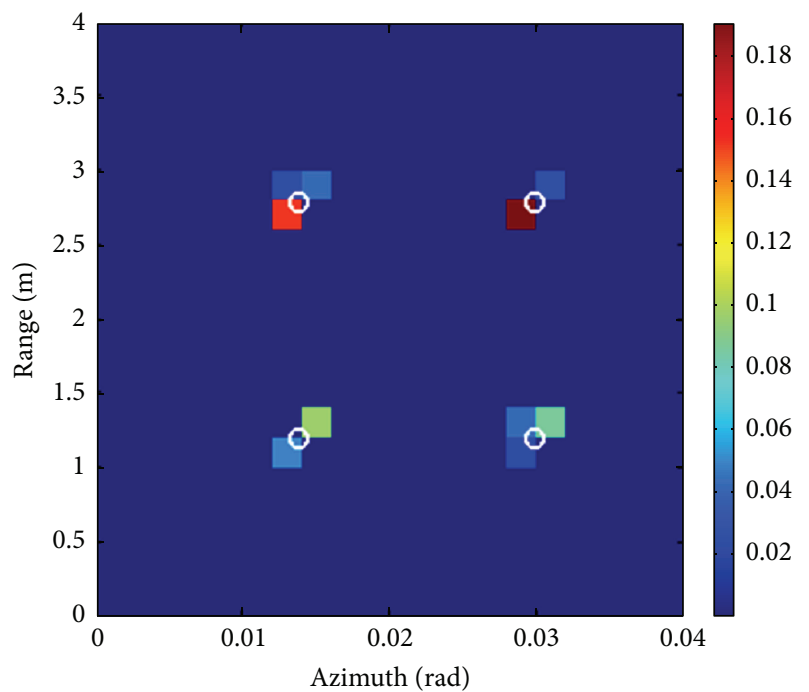

(c)

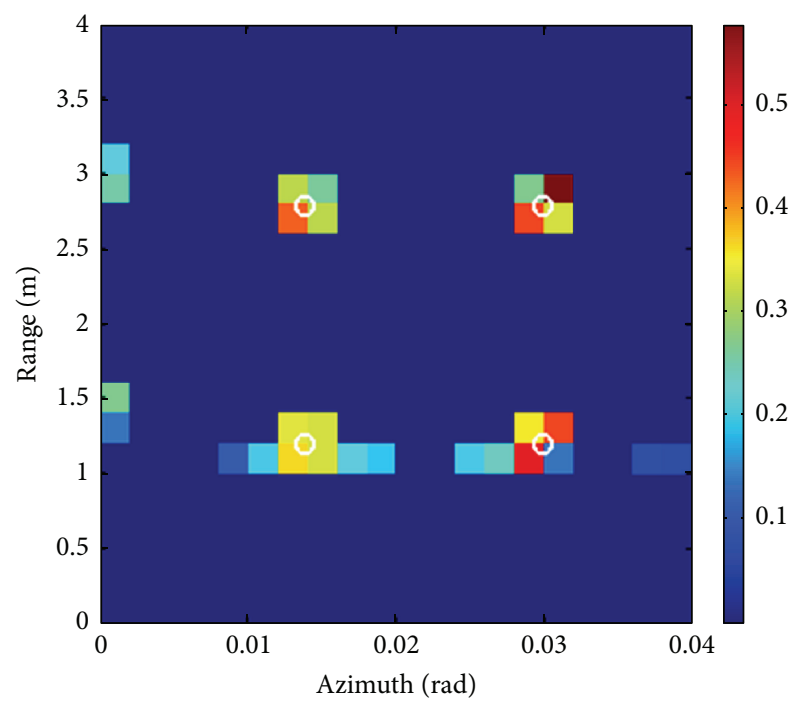

(e)

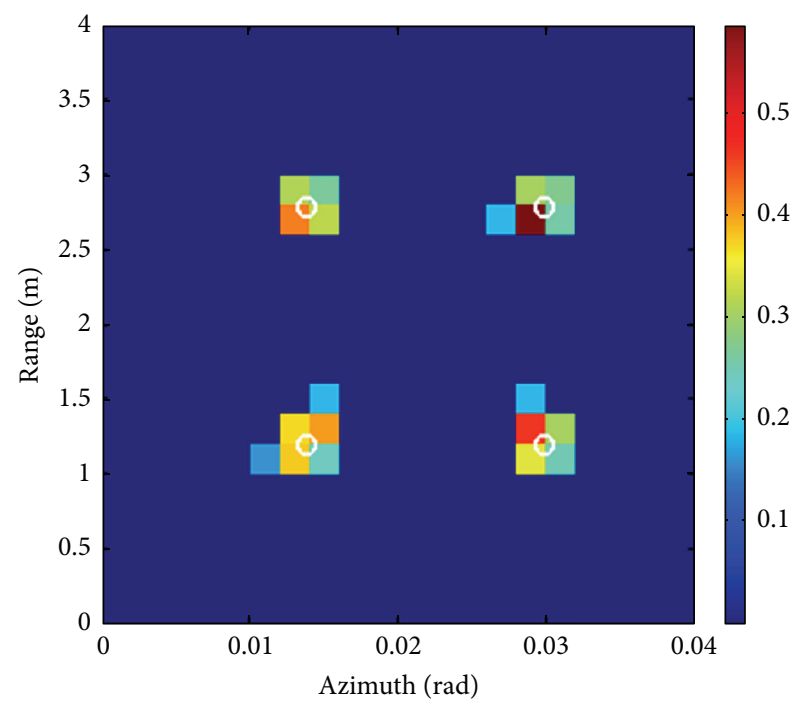

(b)

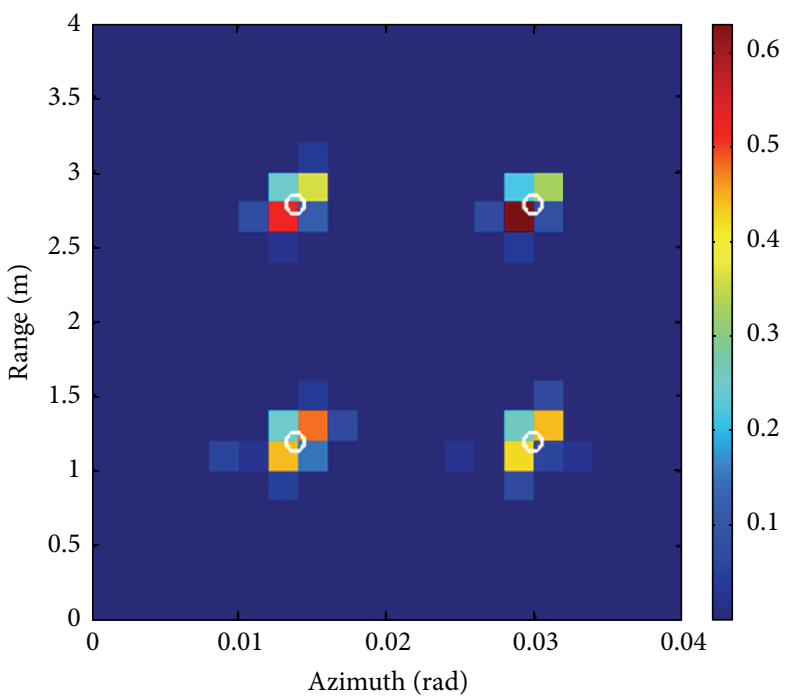

(d)

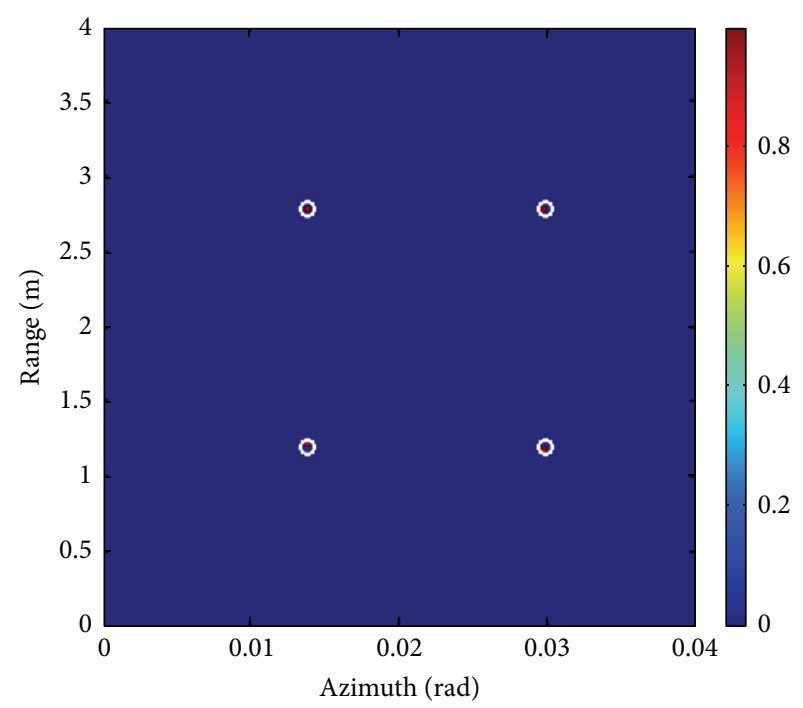

(f)

Figure 8: RCI results when $\zeta=0.61$. (a) MF, (b) OMP, (c) BPDN, (d) S-TLS, (e) CP, and (f) 2D BLOOMP. 


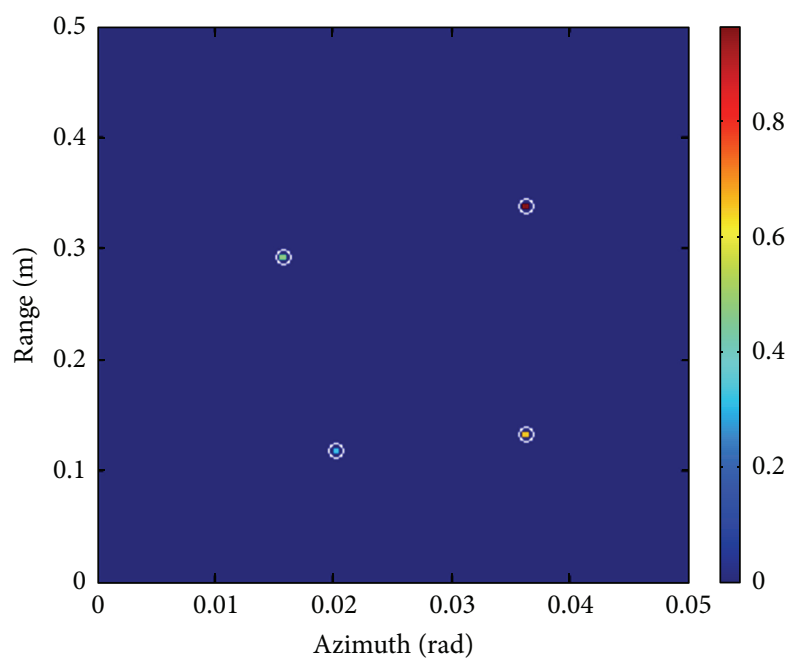

(a)

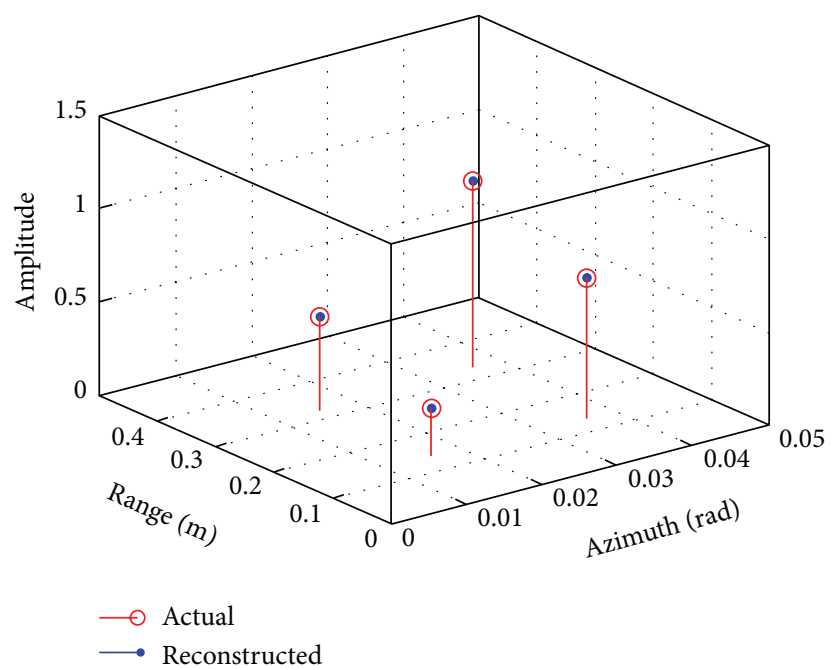

(c)

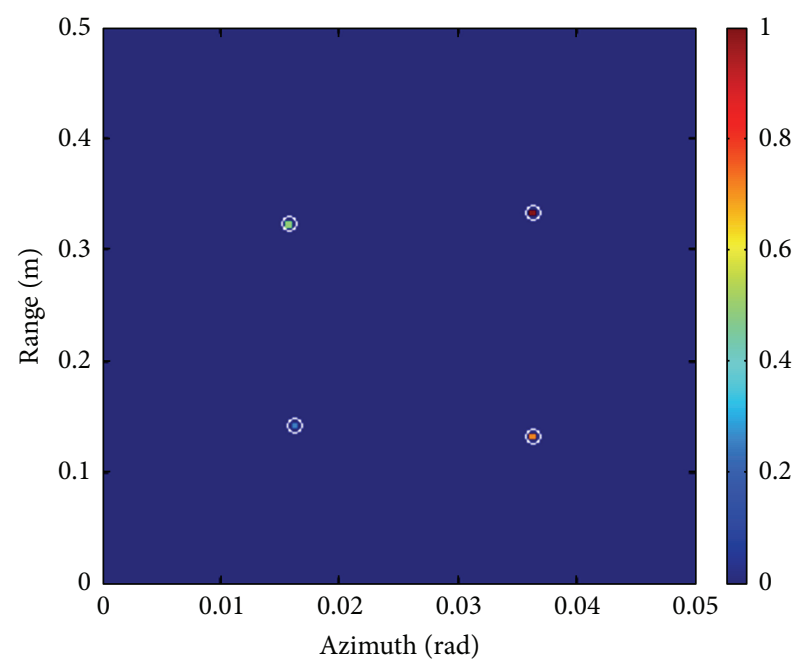

(b)

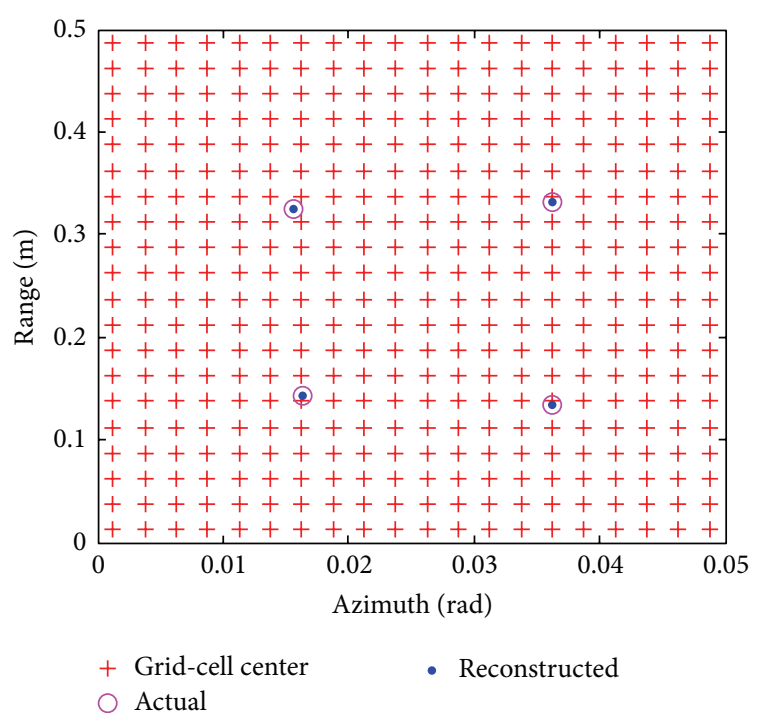

(d)

FIGURE 9: Off-grid RCI results. (a) Reconstructed image by OMP. (b) Reconstructed image by 2D BLOOMP. (c) Result of AIM algorithm. (d) Top view of (c).

Figure 8 shows the reconstructed target image when OGE is 0.61 in the coarse grid. As shown in Figures 6 and 8, $\mathrm{MF}$ is robust to off-grid while the resolution degrades. The target images reconstructed by OMP, BPDN, S-TLS, and CP become worse because of the significant off-grid, while $2 \mathrm{D}$ BLOOMP performs the best among the listed algorithms, as the reconstructed target image is better. However, another problem is raised: "the reason why the performance of $2 \mathrm{D}$ BLOOMP is excellent is the grid refinement?" This is partly true. Precisely speaking, the presented algorithm could realize the sparse recovery in the refinement grid and overcome the effect of coherent dictionary. Moreover, the computation complexity does not increase significantly. CP, BPDN, and S-TLS are time-consuming as the dimension increases with the grid refinement and the performance is not guaranteed. The greedy algorithm, that is, OMP, tends to be much faster than BPDN. However, the coherence of dictionary may not satisfy the requirement due to the grid refinement. Thus, the reconstructed result of OMP may deteriorate significantly, as Figure 9(a) shows.

5.3. Performance of OGE Calibration Algorithm. The numerical experiments show that the grid matching could be realized by the tested algorithms and the scatterers are captured by the nearby grid-cell centers. However, the OGEs are not calibrated, so the imaging performance can be improved further. In Section 4, AIM algorithm is introduced to iteratively calculate the range, azimuth, and scattering-coefficient vector separately. Then, the algorithm is tested to show that the target image is precisely reconstructed based on the grid matching algorithm.

In the simulation, the target space is changed to $0.5 \mathrm{~m} \times$ $0.05 \mathrm{rad}$, and other parameters keep unchanged, comparing 
with the parameter set presented above. Thus, the range grid is much finer than the target space of $4 \mathrm{~m} \times 0.04 \mathrm{rad}$, which will show the performance advantage of BLOOMP more significantly. The OGEs are uniformly distributed within the grid-cell in the refinement grid; that is, $\delta_{\theta k}$ and $\delta_{R k}$ are i.i.d. uniform random variables in $[-0.5,0.5]$.

Figures 9 (a) and $7(\mathrm{~b})$ are the target images reconstructed by OMP and 2D BLOOMP directly in the refinement grids, where the white circles represent the reconstructed scatterers. The actual target scene is shown in Figure 9(d). As shown by the comparison of Figures 9(a) and 9(b), OMP fails in target imaging, while 2D BLOOMP still performs well. We then highlight that the advantage of the presented algorithm is not merely based on the grid refinement. The AIM calibration result is presented in Figures 9(c) and 9(d), where the locations and amplitudes of scatterers are estimated exactly. Moreover, during the experiments, the convergence behavior of AIM algorithm performs well. The relative residual, defined as $\|\overline{\mathbf{A}} \overline{\boldsymbol{\beta}}-\mathbf{y}\|_{2} /\|\mathbf{w}\|_{2}$, reduces rapidly and changes slightly after about merely 9 iterations. In conclusion, the AIM algorithm could converge fast and calibrate the OGE precisely.

\section{Conclusion}

This paper investigates the high-resolution off-grid RCI using $\mathrm{FH}$ waveforms. The off-grid FH-RCI model is set up in the range-azimuth space first. Then, we analyze the off-grid effect on the image quality and conclude that the image quality deteriorates significantly. Furthermore, the CCRB under offgrid is derived based on the MSE of oracle estimator. For offgrid target reconstruction, the process is composed of two stages: grid matching stage and OGE calibration stage, where 2D BLOOMP and AIM algorithms are proposed, respectively. Unlike traditional sparse recovery methods, 2D BLOOMP realizes the recovery in the refinement grids by overwhelming the effect of coherent dictionary and is robust to noise and offgrid. AIM calibration algorithm adaptively adjusts the OGEs and meanwhile seeks the optimal target reconstruction result. Numerical simulations illustrate the validity of the method, which shows the potential for the method to be applied in practical radar systems.

Although only FH-RCI is considered, the framework in this paper can be extended to other imaging radar systems, such as MIMO radar imaging and passive radar imaging. However, the scatterers are assumed to be widely separated to guarantee the imaging in the proposed method, which limits the resolution. Another problem is that some information (i.e., the number of scatterers) should be priorly obtained to improve the performance significantly. Moreover, the imaging results differ for different FH codes; thus, the waveform design is a critical issue which will be investigated in the near future. At last, in the off-grid model formulation, the scatterers and OGEs are jointly sparse and share the common support. Inspired by recent works on block and structured sparsity, our future work is to fully explore the joint sparsity to improve the reconstruction performance.

\section{Competing Interests}

The authors declare that there are no competing interests regarding the publication of this paper.

\section{Acknowledgments}

This work was supported by the National Natural Science Foundation of China (no. 61302149 and no. 61302142) and Research Fund for the Doctoral Program of Higher Education of China (20124307110013).

\section{References}

[1] D. Li, X. Li, Y. Cheng, Y. Qin, and H. Wang, "Radar coincidence imaging: an instantaneous imaging technique with stochastic signals," IEEE Transactions on Geoscience and Remote Sensing, vol. 52, no. 4, pp. 2261-2277, 2014.

[2] D. Li, X. Li, Y. Cheng, Y. Qin, and H. Wang, "Radar coincidence imaging in the presence of target-motion-induced error," Journal of Electronic Imaging, vol. 23, no. 2, Article ID 023014, 2014.

[3] D. Li, X. Li, Y. Cheng, Y. Qin, and H. Wang, "Radar coincidence imaging under grid mismatch," ISRN Signal Processing, vol. 2014, Article ID 987803, 8 pages, 2014.

[4] C.-Y. Chen and P. P. Vaidyanathan, "MIMO radar ambiguity properties and optimization using frequency-hopping waveforms," IEEE Transactions on Signal Processing, vol. 56, no. 12, pp. 5926-5936, 2008.

[5] S. Gogineni and A. Nehorai, "Frequency-hopping code design for MIMO radar estimation using sparse modeling," IEEE Transactions on Signal Processing, vol. 60, no. 6, pp. 3022-3035, 2012.

[6] T. Huang, Y. Liu, H. Meng, and X. Wang, "Cognitive random stepped frequency radar with sparse recovery," IEEE Transactions on Aerospace and Electronic Systems, vol. 50, no. 2, pp. 858870, 2014.

[7] D. L. Donoho, "Compressed sensing," IEEE Transactions on Information Theory, vol. 52, no. 4, pp. 1289-1306, 2006.

[8] E. J. Candes and M. B. Wakin, "An introduction to compressive sampling," IEEE Signal Processing Magazine, vol. 25, no. 2, pp. 21-30, 2008.

[9] X. He, C. Liuc, B. Liu, and D. Wang, "Sparse frequency diverse MIMO radar imaging for off-grid target based on adaptive iterative MAP," Remote Sensing, vol. 5, no. 2, pp. 631-647, 2013.

[10] L. Hu, J.-X. Zhou, Z.-G. Shi, and Q. Fu, "An EM-based approach for compressed sensing using dynamic dictionaries," Journal of Electronics and Information Technology, vol. 34, no. 11, pp. 25542560, 2012.

[11] Y. Chi, L. L. Scharf, A. Pezeshki, and A. R. Calderbank, "Sensitivity to basis mismatch in compressed sensing," IEEE Transactions on Signal Processing, vol. 59, no. 5, pp. 2182-2195, 2011.

[12] X. Han, H. Zhang, and G. Li, "Fast algorithms for sparse recovery withperturbed dictionary," http://arxiv.org/abs/1111.6237.

[13] M. A. Herman and T. Strohmer, "General deviants: an analysis of perturbations in compressed sensing," IEEE Journal on Selected Topics in Signal Processing, vol. 4, no. 2, pp. 342-349, 2010.

[14] M. A. C. Tuncer and A. C. Gurbuz, "Analysis of unknown velocity and target off the grid problems in compressive sensing based subsurface imaging," in Proceedings of the 36th IEEE International Conference on Acoustics, Speech, and Signal Processing (ICASSP '11), pp. 2880-2883, Prague, Czech Republic, May 2011. 
[15] T. L. Hansen, M. A. Badiu, B. H. Fleury, and B. D. Rao, "A sparse Bayesian learning algorithm with dictionary parameter estimation," in Proceedings of the IEEE 8th Sensor Array and Multichannel Signal Processing Workshop, pp. 385-388, IEEE, A Coruña, Spain, June 2014.

[16] A. Fannjiang and H.-C. Tseng, "Compressive radar with off-grid targets: a perturbation approach," Inverse Problems, vol. 29, no. 5, Article ID 054008, 23 pages, 2013.

[17] A. C. Gurbuz, O. Teke, and O. Arikan, "Sparse ground-penetrating radar imaging method for off-the-grid target problem," Journal of Electronic Imaging, vol. 22, no. 2, Article ID 021007, 2013.

[18] D. Malioutov, M. Çetin, and A. S. Willsky, "A sparse signal reconstruction perspective for source localization with sensor arrays," IEEE Transactions on Signal Processing, vol. 53, no. 8, pp. 3010-3022, 2005.

[19] H. Zhu, G. Leus, and G. B. Giannakis, "Sparsity-cognizant total least-squares for perturbed compressive sampling," IEEE Transactions on Signal Processing, vol. 59, no. 5, pp. 2002-2016, 2011.

[20] Z. Tan, P. Yang, and A. Nehorai, "Joint sparse recovery method for compressed sensing with structured dictionary mismatches," IEEE Transactions on Signal Processing, vol. 62, no. 19, pp. 4997-5008, 2014.

[21] Z. Yang, L. Xie, and C. Zhang, "Off-grid direction of arrival estimation using sparse Bayesian inference," IEEE Transactions on Signal Processing, vol. 61, no. 1, pp. 38-43, 2013.

[22] L. Hu, Z. Shi, J. Zhou, and Q. Fu, "Compressed sensing of complex sinusoids: an approach based on dictionary refinement," IEEE Transactions on Signal Processing, vol. 60, no. 7, pp. 38093822, 2012.

[23] G. Tang, B. N. Bhaskar, P. Shah, and B. Recht, "Compressed sensing off the grid," IEEE Transactions on Information Theory, vol. 59, no. 11, pp. 7465-7490, 2013.

[24] C. Ekanadham, D. Tranchina, and E. P. Simoncelli, "Recovery of sparse translation-invariant signals with continuous basis pursuit," IEEE Transactions on Signal Processing, vol. 59, no. 10, pp. 4735-4744, 2011.

[25] A. Fannjiang and W. Liao, "Coherence pattern-guided compressive sensing with unresolved grids," SIAM Journal on Imaging Sciences, vol. 5, no. 1, pp. 179-202, 2012.

[26] Y. Tang, L. Chen, and Y. Gu, "On the performance bound of sparse estimation with sensing matrix perturbation," IEEE Transactions on Signal Processing, vol. 61, no. 17, pp. 4372-4386, 2013.

[27] Z. Ben-Haim and Y. C. Eldar, "The Cramér-Rao bound for estimating a sparse parameter vector," IEEE Transactions on Signal Processing, vol. 58, no. 6, pp. 3384-3389, 2010.

[28] Z. Ben-Haim and Y. C. Eldar, "Near-oracle performance of greedy block-sparse estimation techniques from noisy measurements," IEEE Journal on Selected Topics in Signal Processing, vol. 5, no. 5, pp. 1032-1047, 2011. 


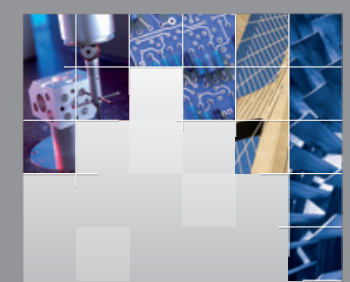

\section{Enfincering}
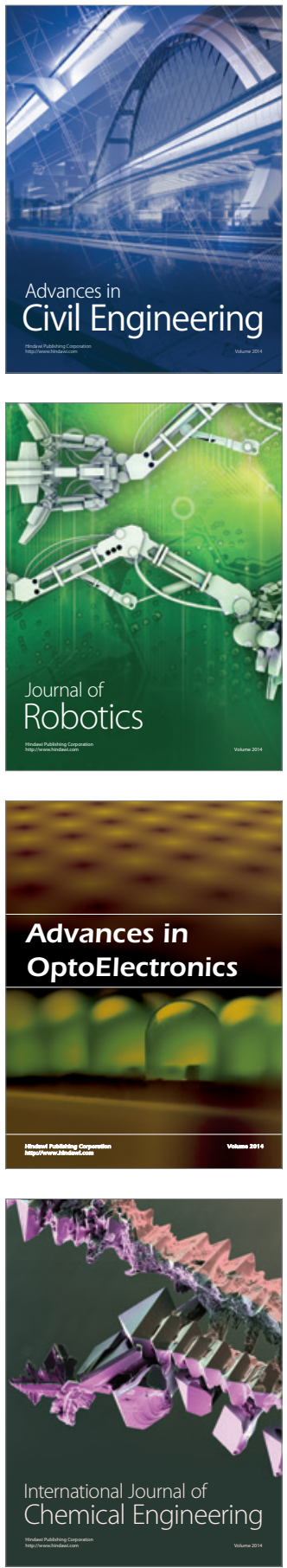

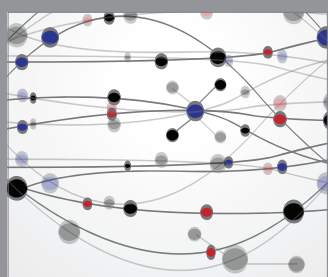

The Scientific World Journal

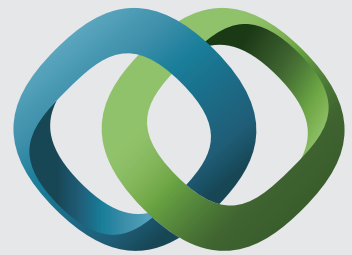

\section{Hindawi}

Submit your manuscripts at

http://www.hindawi.com
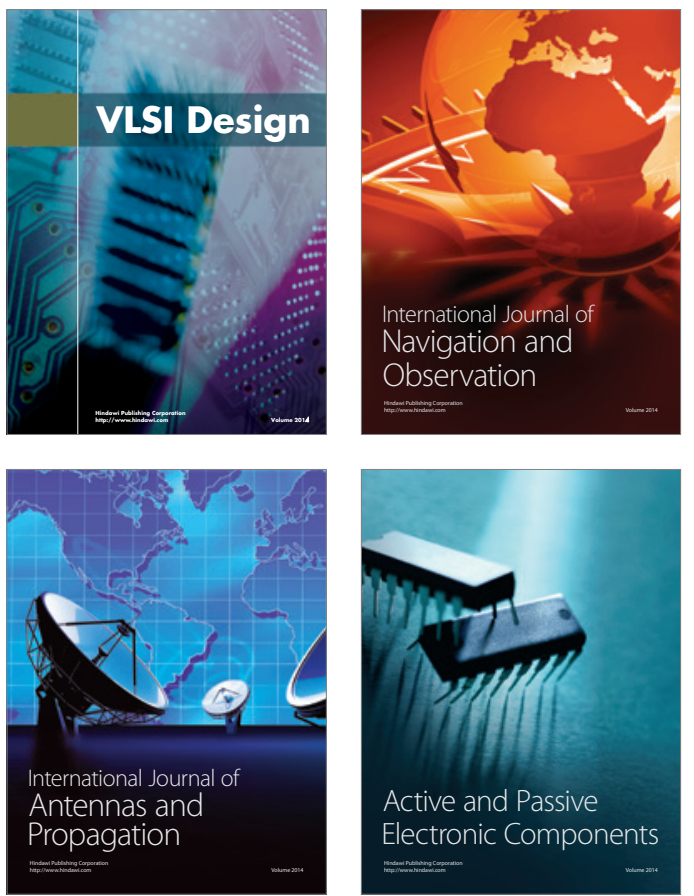
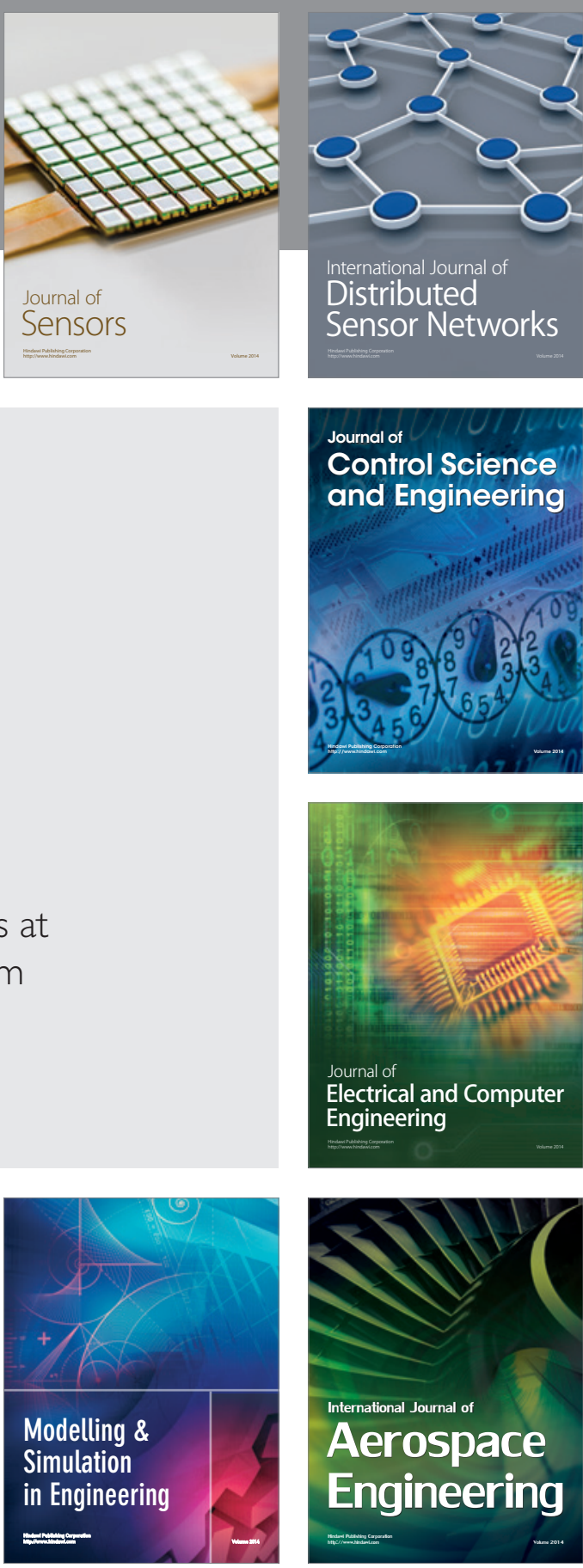

International Journal of

Distributed

Sensor Networks

Journal of

Control Science

and Engineering
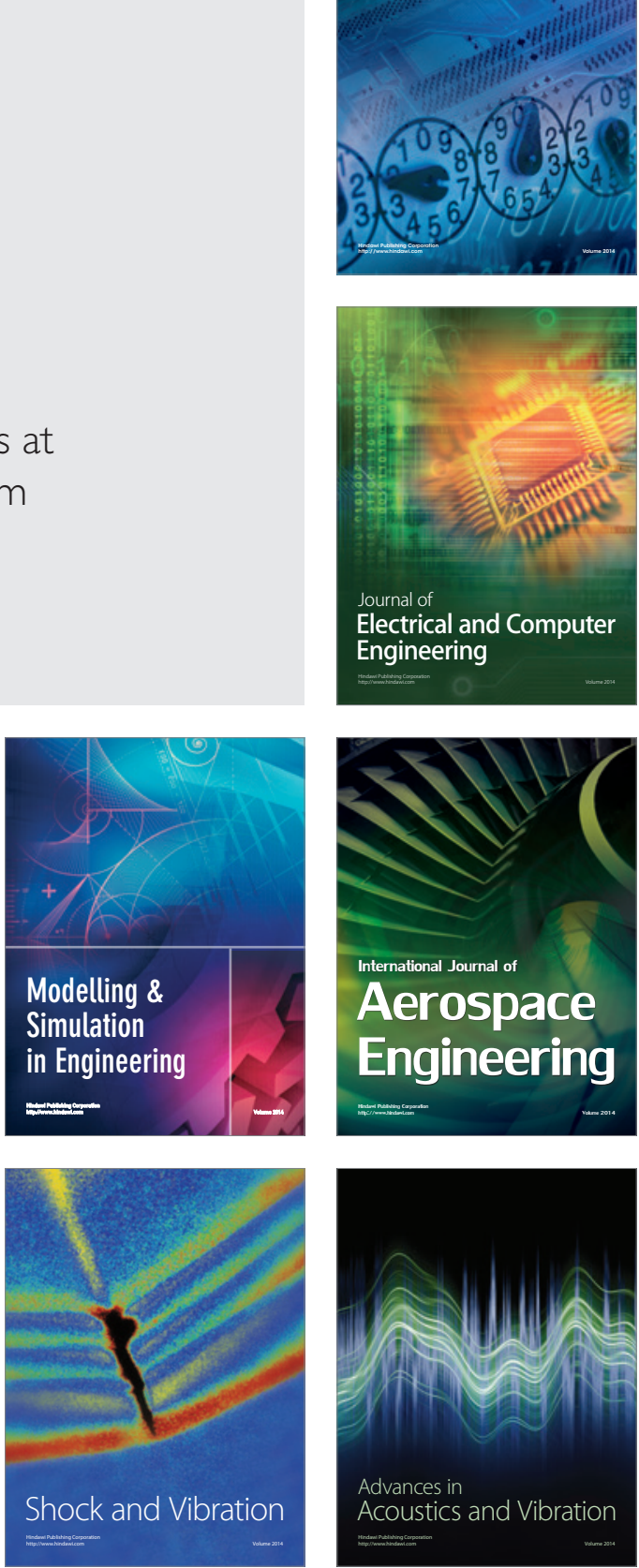